\title{
Contractions of Lie Algebras: Applications to Special Functions and Separation of Variables
}

\author{
E. G. Kalnins \\ Department of Mathematics and Statistics, \\ University of Waikato, \\ Hamilton, New Zealand. \\ W. Miller, Jr. \\ School of Mathematics, University of Minnesota, \\ Minneapolis, Minnesota, 55455, U.S.A. \\ and G. S. Pogosyan* \\ Laboratory of Theoretical Physics, \\ Joint Institute for Nuclear Research, \\ Dubna, Moscow Region 141980, Russia
}

June 17, 1999

\begin{abstract}
We investigate the consequences of contraction of the Lie algebras of the orthogonal groups to the Lie algebras of the Euclidean groups in terms of separation of variables for Laplace-Beltrami eigenvalue equations, and the solutions of these equations that arise through separation of variables techniques, on the $N$-sphere and in $N$-dimensional Euclidean space. General ellipsoidal and paraboloidal coordinates are included, not just the subgroup type coordinates that have been the concern of most investigations of contractions as applied to special functions. We pay special attention to the case

${ }^{*}$ Work supported in part by the Russian Foundation for Basic Research under grant 98-01-00330
\end{abstract}


$N=2$ where we show in detail, for example, how Lamé polynomials contract to periodic Mathieu functions. Our point of view emphasizes the characterization of separable polynomial eigenfunctions in terms of the zeros of these eigenfunctions. We also consider all possible separable coordinate systems on the complex two-sphere (which includes real hyperboloids as special cases) and their contraction to flat space coordinates.

\section{Introduction}

It is well known that contractions of Lie groups and algebras can be used to obtain relations between many of the classical special functions. The most familiar example is perhaps the contraction of the rotation group $S O(3)$ to the Euclidean group $E(2),[1]$. In this example the generators of the Lie algebra of $S O(3)$, which we denote by $L_{k}$, satisfy the commutation relations

$$
\left[L_{i}, L_{j}\right]=\epsilon_{i j k} L_{k}
$$

where $\epsilon_{i j k}$ is the skew symmetric tensor and summation is on the index $k$. An especially clear and comprehensive study of this contraction can be found in the books by Talman and Gilmore $[2,3]$. Consider the matrix element (with respect to the $2 \ell+1$ dimensional irreducible representation) of a group element of $S O(3)$ written in Euler angles as $D^{\ell}(\alpha, \beta, \gamma)_{m n}$, and change $\beta \rightarrow$ $\beta / c$ where $c$ is large and fixed, i.e., $D^{\ell}(\alpha, \beta / c, \gamma)_{m n}=i^{m-n} e^{i m \alpha} d_{m n}^{\ell}(\beta / c) e^{-i n \gamma}$. Now consider the Lie algebra induced using these matrix elements as a basis and the parameters $\alpha, \beta, \gamma$, in which case the basis elements for the Lie algebra are $L_{x}^{\prime}=L_{1} / c, L_{y}^{\prime}=L_{2} / c$ and $L_{z}^{\prime}=L_{3}$. The commutation relations for these new elements are

$$
\left[L_{x}^{\prime}, L_{y}^{\prime}\right]=\frac{1}{c^{2}} L_{z}^{\prime}, \quad\left[L_{y}^{\prime}, L_{z}^{\prime}\right]=L_{x}^{\prime}, \quad\left[L_{z}^{\prime}, L_{x}^{\prime}\right]=L_{y}^{\prime}
$$

In the limit as $c \rightarrow \infty$ these commutators are the same as those of the Lie algebra of the Euclidean group:

$$
\left[P_{1}, P_{2}\right]=0, \quad\left[M, P_{1}\right]=P_{2}, \quad\left[M, P_{2}\right]=-P_{1},
$$

In this limit $L_{z}^{\prime}$ is identified with $M, L_{x}^{\prime}$ with $-P_{2}$ and $L_{y}^{\prime}$ with $P_{1}$. In order for the algebraic relations to have a finite limit we require that the index $\ell$ 
which labels the irreducible representation behaves like $c$ as $c$ becomes large. Specifically we require that $\ell \approx c$. The matrices representing $L_{+}^{\prime}=L_{x}^{\prime}+i L_{y}^{\prime}$ and $L_{-}^{\prime}=L_{x}^{\prime}-i L_{y}^{\prime}$ have elements given by

$$
\begin{aligned}
\left(L_{+}^{\prime}\right)_{m n} & =-\frac{i}{c}[(\ell-n)(\ell+n+1)]^{1 / 2} \delta_{m, n+1}, \\
\left(L_{-}^{\prime}\right)_{m n} & =-\frac{i}{c}[(\ell+n)(\ell-n+1)]^{1 / 2} \delta_{m, n-1} .
\end{aligned}
$$

Then in the limit as $\ell \rightarrow \infty$ the matrix elements of $L_{+}^{\prime}$ and $L_{-}^{\prime}$ assume the form of the matrix elements of $P_{+}$and $P_{-}$in a representation of $E(2)$ labeled by $\chi$. We can take the same limit in the group representations. In this limit one obtains

$$
d_{m n}^{\ell}\left(\frac{\chi \beta}{c}\right) \rightarrow i^{m-n} J_{m-n}(\chi \beta)
$$

as $c \rightarrow \infty$, where $J_{m}(x)$ is a Bessel function [4].

This result is a very special case of the limit procedure for solutions of Laplace-Beltrami eigenvalue equations on the $N$-sphere as the symmetry group of the $N$-sphere, $S O(N+1)$, contracts to the symmetry group $E(N)$ of Euclidean $N$-space. The first investigation of the connection between contractions of the Lie algebras $\mathrm{o}(3)$ and $\mathrm{o}(2,1)$ to $\mathrm{e}(2)$ and separation of variables was done in the articles $[5,6]$. Our objective here is to establish, in detail, just how the contraction procedure works for the various separable coordinates on the two dimensional sphere if the Lie algebra of $S O(3)$ is contracted to that of $E(2)$ and, in general terms, how the procedure works for the $N$-sphere. The analysis of contractions in [2], and in the recent literature, e.g., [7], emphasizes subgroup coordinates. Here we treat the most general separable systems.

\section{Separable coordinates in $\mathbf{N}$ dimensions}

We review the construction of separable coordinates for Laplace-Beltrami eigenvalue equations on the $N$-sphere and $N$-dimensional Euclidean space, $[8,9]$, and show how they are related by contractions.

Elliptic coordinates on the sphere: This is the basic separable coordinate system on the $N$-sphere. Here, $X_{0}, \cdots, X_{N}$ are Cartesian coordinates, 
$e_{0}, \cdots, e_{N}$ are constants and $u_{1}, \cdots, u_{N}$ are elliptic coordinates. The coordinates are related by

$$
\sum_{k=0}^{N} \frac{X_{k}^{2}}{u-e_{k}}=\frac{\prod_{k=1}^{N}\left(u-u_{k}\right)}{\prod_{j=0}^{N}\left(u-e_{j}\right)}, \quad \sum_{k=0}^{N} X_{k}^{2}=1,
$$

where $u$ is a parameter and

$$
\begin{gathered}
e_{0}<u_{1}<e_{1}<u_{2}<\cdots<e_{N-1}<u_{N}<e_{N} . \\
X_{\ell}^{2}=\frac{\prod_{k=1}^{N}\left(e_{\ell}-u_{k}\right)}{\prod_{j \neq \ell}\left(e_{\ell}-e_{j}\right)}, \quad j, \ell=0, \cdots, N .
\end{gathered}
$$

The metric on the sphere is

$$
d s_{e}^{2}=\sum_{\ell=0}^{N} d X_{\ell}^{2}=\sum_{k=1}^{N} \frac{\prod_{m \neq k}\left(u_{m}-u_{k}\right)}{\prod_{\ell=0}^{N}\left(u_{k}-e_{\ell}\right)} d u_{k}^{2} .
$$

The action of the Lie algebra of $S O(N+1)$ on the sphere is given by the operators

$$
L_{k j}=X_{k} \partial_{X_{j}}-X_{j} \partial_{X_{k}}, \quad k, j=0,1, \cdots, N .
$$

The commutation relations are

$$
\left[L_{k j}, L_{q s}\right]=\delta_{j q} L_{k s}-\delta_{k q} L_{j s}-\delta_{j s} L_{k q}+\delta_{k s} L_{j q}, \quad k, j, q, s=0,1, \cdots, N .
$$

The Laplace-Beltrami eigenvalue equation is

$$
\mathcal{I}_{1}^{N} \Psi=-\lambda_{1} \Psi, \quad \mathcal{I}_{1}^{N} \equiv \sum_{i, j} L_{i j}^{2}, \quad \lambda_{1}=\sigma(\sigma+N-1),
$$

where $i, j=0,1, \cdots, N$. A separable solution $\Psi=\prod_{k=1}^{N} \psi_{k}\left(u_{k}\right)$ is characterized by the eigenvalue equations

$$
\mathcal{I}_{k}^{N} \Psi=-\lambda_{k} \Psi, \quad \mathcal{I}_{\ell}^{N}=\sum_{i>j} S_{k}^{(i j)}(e) L_{i j}^{2}, \quad k=1, \cdots, N
$$

where $S_{k}(e)$ are the $k t h$-order elementary symmetric polynomials of $e_{0}, \ldots e_{N}$, or

$$
S_{k}(e)=\left\{\begin{array}{cc}
\sum_{i_{1}>i_{2}>\cdots>i_{k}} e_{i_{1}} \cdots e_{i_{k}} & 1 \leq k \leq N+1 \\
1 & k=0 \\
0 & k>N+1
\end{array}\right.
$$


and $S_{k}^{i j}(e)$ are defined as the $k$ th-order symmetric polynomials of $e_{0}, \ldots e_{j-1}$, $e_{j+1}, \ldots e_{i-1}, e_{i+1}, \ldots e_{N}$.

Here,

$$
\left[\mathcal{I}_{j}^{N}, \mathcal{I}_{k}^{N}\right]=0 .
$$

The separable solutions satisfy the separation equations

$$
-4 \sqrt{\mathcal{P}_{k}} \frac{d}{d u_{k}}\left(\sqrt{\mathcal{P}_{k}} \frac{d \psi_{k}}{d u_{k}}\right)+\left[\sum_{j=1}^{N} \lambda_{j}\left(u_{k}\right)^{N-j}\right] \psi_{k}=0, \quad k=1, \cdots, N
$$

where

$$
\mathcal{P}_{k}=\prod_{q=0}^{N}\left(u_{k}-e_{q}\right)
$$

Elliptic Coordinates in Euclidean space: This is the fundamental separable system in $N$-dimensional Euclidean space. Here, $x_{0}, \cdots, x_{N-1}$ are Cartesian coordinates, $e_{0}, \cdots, e_{N-1}$ are constants and $u_{1}, \cdots, u_{N}$ are elliptic coordinates. The coordinates are related by

$$
\sum_{k=0}^{N-1} \frac{x_{k}^{2}}{u-e_{k}}-c^{2}=-c^{2} \frac{\prod_{k=1}^{N}\left(u-u_{k}\right)}{\prod_{j=0}^{N-1}\left(u-e_{j}\right)}
$$

where

$$
e_{0}<u_{1}<e_{1}<u_{2}<\cdots<e_{N-1}<u_{N}
$$

and

$$
x_{\ell}^{2}=-c^{2} \frac{\prod_{k=1}^{N}\left(e_{\ell}-u_{k}\right)}{\prod_{j \neq \ell}\left(e_{\ell}-e_{j}\right)}, \quad j, \ell=0, \cdots, N-1 .
$$

The metric is

$$
d s_{E}^{2}=\sum_{j=0}^{N-1} d x_{j}^{2}=\frac{c^{2}}{4} \sum_{k=1}^{N} \frac{\prod_{m \neq k}\left(u_{m}-u_{k}\right)}{\prod_{\ell=0}^{N-1}\left(u_{k}-e_{\ell}\right)} d u_{k}^{2} .
$$

The action of the Lie algebra of $E(N)$ on Euclidean space is given by the operators

$$
L_{k j}=x_{k} \partial_{x_{j}}-x_{j} \partial_{x_{k}}, \quad P_{j}=\partial_{x_{j}},
$$

where $k, j=0,1, \cdots, N-1$. The commutation relations are

$$
\left[L_{k j}, L_{q s}\right]=\delta_{j q} L_{k s}-\delta_{k q} L_{j s}-\delta_{j s} L_{k q}+\delta_{k s} L_{j q}
$$




$$
\left[P_{j}, L_{q s}\right]=\delta_{j q} P_{s}-\delta_{j s} P_{q}, \quad k, j, q, s=0,1, \cdots, N-1 .
$$

The Laplace-Beltrami eigenvalue equation is

$$
{ }_{1} \mathcal{J}_{1}^{N} \Psi=-\mu_{1} \Psi, \quad{ }_{1} \mathcal{J}_{1}^{N} \equiv \sum_{j=0}^{N-1} P_{j}^{2} \quad \mu_{1}=k^{2}>0 .
$$

A separable solution $\Psi=\prod_{k=1}^{N} \psi_{k}\left(u_{k}\right)$ is characterized by the eigenvalue equations

$$
{ }_{1} \mathcal{J}_{k}^{N} \Psi=-\mu_{k} \Psi, \quad k=1, \cdots, N
$$

where

$$
{ }_{1} \mathcal{J}_{\ell}^{N}=\sum_{i>j} S_{\ell-2}^{(i j)}(e) L_{i j}^{2}+c^{2} \sum_{i=0}^{N-1} S_{\ell-1}^{(i)}(e) P_{i}^{2}, \quad \ell=2, \cdots, N
$$

and

$$
\left[{ }_{1} \mathcal{J}_{j}^{N},{ }_{1} \mathcal{J}_{k}^{N}\right]=0
$$

The separable solutions satisfy the separation equations

$$
-4 \sqrt{\mathcal{Q}_{k}} \frac{d}{d u_{k}}\left(\sqrt{\mathcal{Q}_{k}} \frac{d \psi_{k}}{d u_{k}}\right)+\left[\sum_{j=1}^{N} \mu_{j}\left(u_{k}\right)^{N-j}\right] \psi_{k}=0, \quad k=1, \cdots, N
$$

where

$$
\mathcal{Q}_{k}=\prod_{q=0}^{N-1}\left(u_{k}-e_{q}\right)
$$

Parabolic coordinates in Euclidean space: This is a second fundamental separable system in $N$-dimensional Euclidean space, though as we shall show, it can be obtained by contraction from elliptic coordinates in Euclidean space. Here, $y_{0}, \cdots, y_{N-1}$ are Cartesian coordinates, $e_{1}, \cdots, e_{N-1}$ are constants and $u_{1}, \cdots, u_{N}$ are parabolic coordinates. The coordinates are related by

$$
\begin{gathered}
-2 c y_{0}-c^{2} u+\sum_{k=1}^{N-1} \frac{y_{k}^{2}}{u-e_{k}}=-c^{2} \frac{\prod_{k=1}^{N}\left(u-u_{k}\right)}{\prod_{j=1}^{N-1}\left(u-e_{j}\right)}, \\
y_{0}=\frac{c}{2}\left(-\sum_{j=1}^{N} u_{j}+\sum_{k=1}^{N-1} e_{k}\right) \\
y_{i}^{2}=-c^{2} \frac{\prod_{j=1}^{N}\left(u_{j}-e_{i}\right)}{\prod_{k \neq i}\left(e_{k}-e_{i}\right)}, \quad i, k=1, \cdots, N-1, \quad j=1, \cdots, N,
\end{gathered}
$$


where

$$
u_{1}<e_{1}<u_{2}<e_{2}<\cdots<e_{N-1}<u_{N}
$$

The metric is

$$
d s_{P}^{2}=\sum_{\ell=0}^{N-1} d y_{\ell}^{2}=\frac{c^{2}}{4} \sum_{k=1}^{N} \frac{\prod_{m \neq k}\left(u_{m}-u_{k}\right)}{\prod_{\ell=1}^{N-1}\left(u_{k}-e_{\ell}\right)} d u_{k}^{2} .
$$

The action of $E(N)$ is given by (18), with $x_{i}$ replaced everywhere by $y_{i}$.

The Laplace-Beltrami eigenvalue equation is

$$
{ }_{2} \mathcal{J}_{1}^{N} \Psi=-\rho_{1} \Psi, \quad{ }_{2} \mathcal{J}_{1}^{N} \equiv \sum_{j=0}^{N-1} P_{j}^{2}, \quad \rho_{1}=k^{2}>0 .
$$

A separable solution $\Psi=\prod_{k=1}^{N} \psi_{k}\left(u_{k}\right)$ is characterized by the eigenvalue equations

$$
{ }_{2} \mathcal{J}_{k}^{N} \Psi=-\rho_{k} \Psi, \quad k=1, \cdots, N
$$

where

$$
\begin{array}{r}
{ }_{2} \mathcal{J}_{\ell}^{N}=-\sum_{i>j>0} S_{\ell-3}^{(i j)}(e) L_{i j}^{2}+c^{2} \sum_{i=1}^{N-1} S_{\ell-1}^{(i)}(e) P_{i}^{2}+c \sum_{j=1}^{N-1} S_{\ell-2}^{(j)}(e)\left\{P_{j}, L_{j 0}\right\}_{+} \\
+c^{2} S_{\ell-1}(e) P_{0}^{2}, \quad \ell=2, \cdots, N
\end{array}
$$

and $\{A, B\}_{+}=A B+B A$. The sums $S_{k}(e)$ are defined as before, except that $S_{-1}^{(i j)}(e)=0, e_{0}=0$. We have

$$
\left[{ }_{2} \mathcal{J}_{j}^{N},{ }_{2} \mathcal{J}_{k}^{N}\right]=0
$$

The separable solutions satisfy the separation equations

$$
-4 \sqrt{\mathcal{H}_{k}} \frac{d}{d u_{k}}\left(\sqrt{\mathcal{H}_{k}} \frac{d \psi_{k}}{d u_{k}}\right)+\left[\sum_{j=1}^{N} \rho_{j}\left(u_{k}\right)^{N-j}\right] \psi_{k}=0, \quad k=1, \cdots, N
$$

where

$$
\mathcal{H}_{k}=\prod_{q=1}^{N-1}\left(u_{k}-e_{q}\right)
$$


Elliptic coordinates on sphere $\Rightarrow$ Elliptic coordinates in Euclidean space: We describe in detail how one obtains elliptic coordinates in Euclidean space from elliptic coordinates on the sphere, via contraction. Let, as usual, the Cartesian coordinates on the sphere be denoted

$$
\left(X_{0}, \cdots, X_{N}\right), \quad \sum_{\ell=0}^{N} X_{\ell}^{2}=1
$$

and set the inhomogeneous coordinates [5]

$$
x_{j}^{2}=R c^{2} \frac{X_{j}^{2}}{X_{N}^{2}}, \quad j=0, \cdots, N-1 .
$$

Let $R=e_{N} \rightarrow+\infty$. Then $X_{N} \rightarrow 1$ and

$$
\begin{gathered}
x_{j}^{2}(R) \rightarrow-c^{2} \frac{\prod_{k=1}^{N}\left(e_{j}-u_{k}\right)}{\prod_{\ell \neq j}\left(e_{j}-e_{\ell}\right)}, \quad j, \ell=0, \cdots, N-1, \\
d s_{E}^{2}=-\frac{c^{2}}{4} \lim _{R=e_{N} \rightarrow \infty} R d s_{e}^{2}\left(e_{N}\right) .
\end{gathered}
$$

This amounts to letting the radius $r=\sqrt{R}$ of the $N$-sphere and a focus go to $\infty$. (Similarly, can take the limit $R=-e_{0} \rightarrow+\infty$ with $X_{0} \rightarrow 1$ to get Euclidean elliptic coordinates $\left\{e_{\ell}^{\prime}, u_{j}^{\prime}\right\}$ with $e_{\ell}^{\prime}=-e_{N-\ell}, u_{j}^{\prime}=-u_{N-j+1}$, $\ell=0, \cdots, N-1$ and $j=1, \cdots, N$.)

Making the change of coordinates (35), we see that the Lie algebra action transforms as follows:

$$
\begin{aligned}
L_{N s}=X_{N} \partial_{X_{s}}-X_{s} \partial_{X_{N}} & =c \sqrt{R} \partial_{x_{s}}+\frac{x_{s}}{c \sqrt{R}} \sum_{j=0}^{N-1} x_{j} \partial_{x_{j}} \\
L_{k s}=X_{k} \partial_{X_{s}}-X_{s} \partial_{X_{k}} & =x_{k} \partial_{x_{s}}-x_{s} \partial_{x_{k}}, \quad s, k=0, \cdots, N-1 .
\end{aligned}
$$

Now we set $P_{s}^{\prime}=\lim _{R \rightarrow \infty} \frac{1}{c \sqrt{R}} L_{N s}, L_{k s}^{\prime}=L_{k s}$ and verify that, in the limit, the primed operators and their commutation relations agree with (18) - (20) for the action of $E(N)$. Furthermore, one can easily verify that

$$
\mathcal{I}_{1}^{\prime N} \equiv \lim _{R \rightarrow \infty} \frac{1}{c^{2} R} \mathcal{I}_{1}^{N}={ }_{1} \mathcal{I}_{1}^{N}, \quad \mathcal{I}_{k}^{\prime N} \equiv \lim _{R \rightarrow \infty} \frac{1}{R} \mathcal{I}_{k}^{N}={ }_{1} \mathcal{I}_{k}^{N}, \quad k=2, \cdots, N
$$


Thus the operators defining separation in elliptic coordinates on the sphere go in the limit to the operators defining separation in elliptic coordinates on Euclidean space.

Now suppose $\Psi$ is a separable solution on the sphere, i.e., it satisfies eigenvalue equations (11). Then if we consider a one-parameter family $\Psi(R)$ of solutions such that $\lambda_{1} \approx c^{2} R \mu_{1}, \lambda_{k} \approx R \mu_{k}, k=2, \cdots, N$, and $\Psi^{\prime}=$ $\lim _{R=e_{n} \rightarrow \infty} \Psi(R)$ exists and is non-zero, it follows that $\Psi^{\prime}$ satisfies the eigenvalue equations (22) for separation in elliptic coordinates on Euclidean space.

Elliptic coordinates in Euclidean space $\Rightarrow$ Parabolic coordinates: Now we describe how to obtain parabolic coordinates in Euclidean space from elliptic coordinates in Euclidean space, via contraction.

Set

$$
\begin{aligned}
c^{2}\left(R-\frac{y_{0}}{c}\right)^{2} & =-R c^{2} \frac{\prod_{j=1}^{N}\left(e_{0}-u_{j}\right)}{\prod_{k=1}^{N-1}\left(e_{0}-e_{k}\right)} \\
y_{j}^{2} & =R c^{2} \frac{\prod_{k=1}^{N}\left(u_{k}-e_{j}\right)}{\prod_{\ell \neq j}\left(e_{\ell}-e_{j}\right)}, \\
j=1, \cdots, N-1, \quad k & =1, \cdots, N, \quad \ell=0, \cdots, N-1,
\end{aligned}
$$

i.e., set

$$
y_{0}=c R-\sqrt{R} x_{0}, \quad y_{k}=\sqrt{R} x_{k}, \quad k=1, \cdots, N-1
$$

where the $x_{i}$ are cartesian coordinates (18), related to elliptic coordinates via (16).

Let $R=-e_{0} \rightarrow+\infty$. Then, in the limit, (41) and (42) yield, respectively,

$$
\begin{aligned}
-2 c y_{0} & =c^{2}\left(\sum_{k=1}^{N} u_{k}-\sum_{j=1}^{N-1} e_{j}\right) \\
y_{j}^{2} & =-c^{2} \frac{\prod_{k=1}^{N}\left(u_{k}-e_{j}\right)}{\prod_{k \neq j}\left(e_{k}-e_{j}\right)} . \\
d s_{P}^{2} & =\lim _{R=-e_{0} \rightarrow \infty} R d s_{E}^{2}\left(e_{0}\right) .
\end{aligned}
$$

Furthermore, from (43) we have

$$
L_{\ell k}=x_{\ell} \partial_{x_{k}}-x_{k} \partial_{x_{\ell}}=y_{\ell} \partial_{y_{k}}-y_{k} \partial_{y_{\ell}}
$$




$$
\begin{gathered}
L_{0 k}=x_{0} \partial_{x_{k}}-x_{k} \partial_{x_{0}}=-y_{o} \partial_{y_{k}}+y_{k} \partial_{y_{0}}+c R \partial_{y_{k}}, \\
P_{0}=\partial_{x_{0}}=-\sqrt{R} \partial_{y_{0}}, \quad P_{k}=\partial_{x_{k}}=\sqrt{R} \partial_{y_{k}}, \quad k, \ell=1, \cdots, N-1 .
\end{gathered}
$$

Now we define new operators by

$$
\begin{aligned}
& L_{\ell k}^{\prime}=L_{\ell k}, \quad L_{0 k}^{\prime}=\lim _{R=-e_{0} \rightarrow \infty}\left(L_{0 k}-c \sqrt{R} P_{k}\right), \\
& P_{k}^{\prime}=\lim _{R=-e_{0} \rightarrow \infty} \frac{P_{k}}{\sqrt{R}}, \quad P_{0}^{\prime}=-\lim _{R=-e_{0} \rightarrow \infty} \frac{P_{0}}{\sqrt{R}},
\end{aligned}
$$

and see that in the limit these operators satisfy (18).

Also, one can easily verify that

$$
{ }_{1} \mathcal{J}^{\prime N} \equiv \lim _{R=-e_{0} \rightarrow \infty} \frac{1}{R} \mathcal{I}_{k}^{N}={ }_{2} \mathcal{J}_{k}^{N}, \quad k=1, \cdots, N .
$$

Thus the operators defining separation in elliptic coordinates on Euclidean space go in the limit to the operators defining separation in parabolic coordinates on Euclidean space.

Now suppose $\Psi$ is a separable solution in Euclidean elliptic coordinates, i.e., it satisfies eigenvalue equations (22). Then if we consider a one-parameter family $\Psi(R)$ of solutions such that $\mu_{k} \approx R \rho_{k}, k=1, \cdots, N$, and $\Psi^{\prime}=$ $\lim _{R=-e_{0} \rightarrow \infty} \Psi(R)$ exists and is non-zero, it follows that $\Psi^{\prime}$ satisfies the eigenvalue equations (31) for separation in parabolic coordinates on Euclidean space.

\section{Hybrid separable coordinate systems}

A complete description of separable coordinate systems on the $N$-sphere and on Euclidean $N$-space, and a graphical method for constructing these systems can be found in $[8,9]$. Here we mention some of the main ideas.

The basic elliptic coordinate system on the $N$-sphere is denoted

$$
\left[e_{0}\left|e_{1}\right| \cdots \mid e_{N}\right] .
$$

All separable coordinate systems on the $N$-sphere can be obtained by nesting these basic coordinates for the $k$-spheres for $k \leq N$. For example we can obtain a separable coordinate system on the $N$-sphere by starting with a 
basic elliptic coordinate system on the $(N-k)$-sphere and embedding in it a $k$-sphere. The $k$-sphere Cartesian coordinates $\left(V_{0}, \cdots, V_{k}\right)$ can be attached to any one of the $N-k+1$ Cartesian coordinates $\left(U_{0}, \cdots, U_{N-k}\right)$ of the $(N-k)$-sphere. Let us attach it to the first coordinate. Then we have

$$
\begin{gathered}
\left(X_{0}, \cdots, X_{N}\right)=\left(U_{0} V_{0}, \cdots, U_{0} V_{k}, U_{1}, \cdots, U_{N-k}\right), \quad \sum_{\ell=0}^{k} V_{\ell}^{2}=1, \\
V_{\ell}^{2}=\frac{\prod_{i=1}^{k}\left(v_{i}-f_{\ell}\right)}{\prod_{i \neq \ell}\left(f_{i}-f_{\ell}\right)}, \quad U_{0}^{2}=\frac{\prod_{t=1}^{N-k}\left(u_{t}-e_{0}\right)}{\prod_{i \neq 0}\left(e_{i}-e_{0}\right)} \\
d s^{2}=d s_{1}^{2}+U_{0}^{2} d s_{2}^{2}, \quad d s_{1}^{2}=\sum_{h=0}^{N-k} d U_{h}^{2}, \quad d s_{2}^{2}=\sum_{\ell=0}^{k} d V_{\ell}^{2} .
\end{gathered}
$$

The resulting system is denoted graphically by

$$
\begin{aligned}
& {\left[\begin{array}{c|c|c|c}
e_{0} & \mid e_{1} & \cdots & e_{N-k}
\end{array}\right]} \\
& \downarrow \\
& {\left[\begin{array}{c|c|c}
f_{0} & \cdots & f_{k}
\end{array}\right]}
\end{aligned}
$$

Here is another possibility:

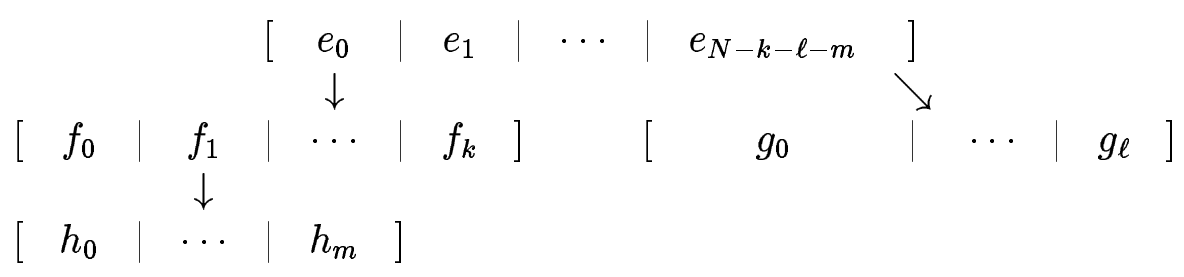

Each separable system can be obtained in this way via embeddings. The graph is a tree whose nodes are basic elliptic coordinate systems.

For Euclidean space the results are a bit more complicated. The basic ellipsoidal coordinate system on $N$-space is denoted

$$
<e_{0}\left|e_{1}\right| \cdots \mid e_{N-1}>
$$

and the parabolic coordinate system is

$$
\left(e_{1}|\cdots| e_{N-1}\right)
$$

The graphs need no longer be trees; they can have several connected components. Each connected component is a tree with a root node that is either of 
the form (55) or (56). Just as above, spheres (49) can be embedded in the root coordinates or to each other. Here are two examples:

$$
<e_{0}>\quad<e_{0}^{\prime}>
$$

1) Cartesian coordinates in two-space, and 2) oblate spheroidal coordinates

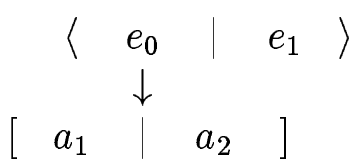

in three-space.

Procedure for contractions from general separable coordinates on the $N$-sphere (labeled by a tree) to ellipsoidal type coordinates in Euclidean $N$-space:

1) Go to the root of the tree.

$$
\left[e_{0}\left|e_{1}\right| \cdots \mid e_{k-1}\right]
$$

2) Erase either the left-hand square or the right-hand square in this $k$ square block. The resulting $(k-1)$-block becomes a diamond block, say

$$
<e_{1}|\cdots| e_{k-1}>
$$

denoting ellipsoidal coordinates in Euclidean space.

3a) If the erased square is not connected to some lower block, the process ends.

$3 \mathrm{~b}$ ) If the erased square is connected to a lower block, erase the edge, proceed to the lower block and repeat step 2).

When the process ends we have a coordinate system in Euclidean space with one more component than the number of edges erased.

For example, one result of contracting (53), in particular letting $R=$ $e_{N-k} \rightarrow \infty$, is to obtain the Euclidean $N$-space coordinate system

$$
\begin{aligned}
& \left\langle\begin{array}{l|l|l|l}
e_{0} & e_{1} & \cdots & e_{N-k-1}
\end{array}\right\rangle \\
& \downarrow \\
& {\left[\begin{array}{l|l|l}
f_{0} & \cdots & f_{k}
\end{array}\right]}
\end{aligned}
$$


We treat another example in detail, a coordinate system on the 6-sphere:

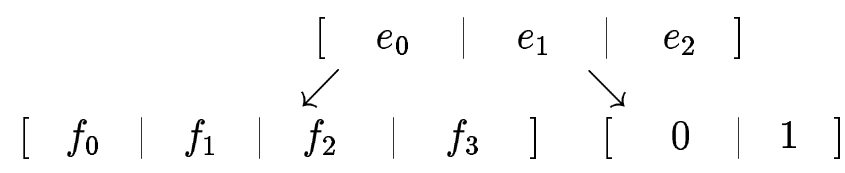

The presciption for writing down the invariant operators corresponding to embedded coordinate systems can be found in $[8,9]$. The results in this case are

$$
\begin{aligned}
& \mathcal{I}_{1}=\sum_{i>j} L_{i j}^{2}, \quad 0 \leq i, j \leq 6 \\
& \mathcal{I}_{2}=e_{0}\left(L_{64}^{2}+L_{65}^{2}\right)+e_{1} \sum_{i=0}^{3} L_{6 i}^{2}+e_{2} \sum_{i=0}^{3}\left(L_{4 i}^{2}+L_{5 i}^{2}\right), \\
& \mathcal{I}_{3}=\sum_{k>\ell} L_{k \ell}^{2}, \quad 0 \leq k, \ell \leq 3 \\
& \mathcal{I}_{4}=L_{01}^{2}\left(f_{2}+f_{3}\right)+L_{02}^{2}\left(f_{1}+f_{3}\right)+L_{03}^{2}\left(f_{1}+f_{2}\right)+L_{12}^{2}\left(f_{0}+f_{3}\right) \\
& \quad+L_{13}^{2}\left(f_{0}+f_{2}\right)+L_{23}^{2}\left(f_{0}+f_{1}\right), \\
& \mathcal{I}_{5}=L_{01}^{2} f_{2} f_{3}+L_{03}^{2} f_{1} f_{2}+L_{12}^{2} f_{0} f_{3}+L_{13}^{2} f_{0} f_{2}+L_{23}^{2} f_{0} f_{1}, \\
& \mathcal{I}_{6}=L_{45}^{2} .
\end{aligned}
$$

In the limit as $R=e_{2} \rightarrow \infty$ with eigenvalues $\lambda_{j}=R \mu_{j}, j=1,2$ and $\lambda_{k}=\mu_{k}$, $3 \leq k \leq 6$, we get the coordinate system in Euclidean 6-space

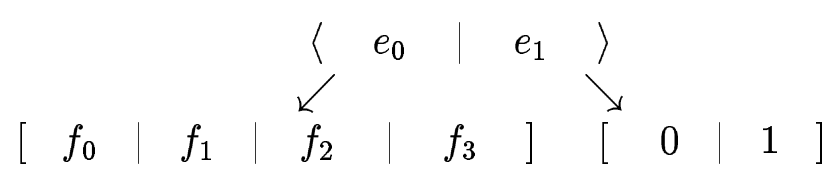

with invariant operators

$$
\begin{aligned}
& \mathcal{I}_{1}^{\prime}=\sum_{i=0}^{5} P_{i}^{2}, \\
& \mathcal{I}_{2}^{\prime}=c^{2} e_{0}\left(P_{4}^{2}+P_{5}^{2}\right)+c^{2} e_{1} \sum_{i=0}^{3} P_{i}^{2}+\sum_{i=0}^{3}\left(L_{4 i}^{2}+L_{5 i}^{2}\right), \\
& \mathcal{I}_{k}^{\prime}=I_{k}, \quad 3 \leq k \leq 6 .
\end{aligned}
$$




\section{The real two-sphere}

To see in detail how this contraction works on the two-sphere, and its relation to special functions, we can specialise the Bessel function example to the case when $n=0$ so that the limit (4) has the form $[1,5]$

$$
P_{\ell m}\left(\cos \left(\frac{\chi \beta}{c}\right)\right) \rightarrow i^{m} J_{m}(\chi \beta)
$$

to within a suitable normalisation, where $P_{\ell m}(x)$ is an associated Legendre polynomial. (For general $n$ one needs to employ the Laplace-Beltrami operator on the three-sphere [7].) Spherical coordinates on the two-sphere are given by

$$
\begin{aligned}
& s_{1}^{2}=\frac{\left(u_{1}-e_{0}\right)\left(u_{2}-a_{0}\right)}{\left(e_{1}-e_{0}\right)\left(a_{1}-a_{0}\right)}, \quad s_{2}^{2}=\frac{\left(u_{1}-e_{0}\right)\left(u_{2}-a_{1}\right)}{\left(e_{1}-e_{0}\right)\left(a_{0}-a_{1}\right)} \\
& s_{3}^{2}=\frac{\left(u_{1}-e_{1}\right)}{\left(e_{0}-e_{1}\right)}
\end{aligned}
$$

where $e_{0}<u_{1}<e_{1}$, and $a_{0}<u_{2}<a_{1}$. Here $s_{1}^{2}+s_{2}^{2}+s_{3}^{2}=1$. This can be recognised as the rational form of the normal spherical coordinates. Indeed if we were to make transformations of the form $u \rightarrow \alpha u+\beta$ we could define new variables $v_{1}, v_{2}$ and effectively take $0<v_{1}<1,0<v_{2}<1$. The variables $v_{1}, v_{2}$ could then be identified as $v_{1}=\sin ^{2} \theta$ and $v_{2}=\cos ^{2} \varphi$, the normal spherical coordinates. The way we have defined spherical coordinates in this case corresponds to the choice of graph

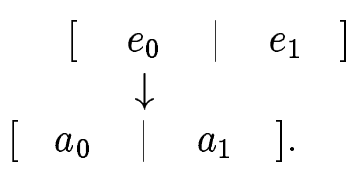

The corresponding Laplace-Beltrami eigenvalue equation has the form

$$
\begin{gathered}
{\left[4\left(u_{1}-e_{0}\right)\left(u_{1}-e_{1}\right)\left[\partial_{u_{1}}^{2}+\frac{1}{2}\left(\frac{2}{u_{1}-e_{0}}+\frac{1}{u_{1}-e_{1}}\right) \partial_{u_{1}}\right]+\right.} \\
\left.\frac{e_{0}-e_{1}}{u_{1}-e_{0}}\left(u_{2}-a_{0}\right)\left(u_{2}-a_{1}\right)\left[\partial_{u_{2}}^{2}+\frac{1}{2}\left(\frac{1}{u_{2}-a_{0}}+\frac{1}{u_{2}-a_{1}}\right) \partial_{u_{2}}\right]+\ell(\ell+1)\right] \Psi=0 .
\end{gathered}
$$


If we now make the requirement that $e_{1} \approx \ell^{2} / \chi^{2}$ as $\ell \rightarrow \infty$ then the corresponding equation looks like

$$
\begin{gathered}
{\left[4\left(u_{1}-e_{0}\right)\left[\partial_{u_{1}}^{2}+\frac{1}{u_{1}-e_{0}} \partial_{u_{1}}\right]+\right.} \\
\left.\frac{1}{u_{1}-e_{0}}\left(u_{2}-a_{0}\right)\left(u_{2}-a_{1}\right)\left[\partial_{u_{2}}^{2}+\frac{1}{2}\left(\frac{1}{u_{2}-a_{0}}+\frac{1}{u_{2}-a_{1}}\right) \partial_{u_{2}}\right]+\chi^{2}\right] \Psi=0
\end{gathered}
$$

In both cases $\Psi$ can be written as $\Phi A\left(u_{2}\right)$ where $A\left(u_{2}\right)$ satisfies

$$
\left[\left(u_{2}-a_{0}\right)\left(u_{2}-a_{1}\right)\left(\partial_{u_{2}}^{2}+\frac{1}{2}\left(\frac{1}{u_{2}-a_{0}}+\frac{1}{u_{2}-a_{1}}\right)\right) \partial_{u_{2}}\right] A\left(u_{2}\right)=-m^{2} A\left(u_{2}\right) .
$$

In the case of coordinates on the sphere the resulting separation equation for $\Phi$ is

$$
\begin{gathered}
{\left[4\left(u_{1}-e_{0}\right)\left(u_{1}-e_{1}\right)\left[\partial_{u_{1}}^{2}+\frac{1}{2}\left(\frac{2}{u_{1}-e_{0}}+\frac{1}{u_{1}-e_{1}}\right) \partial_{u_{1}}\right]\right.} \\
\left.-\frac{e_{0}-e_{1}}{u_{1}-e_{0}} m^{2}+\ell(\ell+1)\right] \Phi=0 .
\end{gathered}
$$

In the limit as $e_{1} \approx \ell^{2} / \chi^{2}$ and $\ell \rightarrow \infty$ the corresponding equation becomes

$$
\left[4\left(u_{1}-e_{0}\right)\left[\partial_{u_{1}}^{2}+\frac{1}{u_{1}-e_{0}} \partial_{u_{1}}\right]-\frac{m^{2}}{u_{1}-e_{0}}+\chi^{2}\right] \Psi=0
$$

This is a form of Bessel's equation [4]. To establish the basic mechanism for separation we examine the equation for $\Phi$. If we consider the separation equation and write $\Phi=\left(u_{1}-e_{0}\right)^{m / 2} \Lambda\left(u_{1}\right)$ then the equation for $\Lambda$ is

$$
\begin{gathered}
\left\{4\left(u_{1}-e_{0}\right)\left(u_{1}-e_{1}\right) \partial_{u_{1}}^{2}+\right. \\
\left.\left[u_{1}(6+4 m)-2 e_{0}-4 e_{1}(m+1)\right] \partial_{u_{1}}-(\ell-m)(\ell+m+1)\right\} \Lambda=0 .
\end{gathered}
$$

If we look for a polynomial solution for $\Lambda$ of the form $\Lambda=\Pi_{h=1}^{q}\left(u_{1}-\theta_{h}\right)$ we see that we have a solution if and only if the zeros $\theta_{h}$ satisfy the fundamental relation $[10,11]$

$$
\frac{2(m+1)}{\theta_{j}-e_{0}}+\frac{1}{\theta_{j}-e_{1}}+\sum_{k \neq j} \frac{4}{\theta_{j}-\theta_{k}}=0 .
$$


This is the familiar form of the equations determining the zeros of the generalised Legendre polynomials. In order to determine how the polynomial behaves as $\ell, e_{1} \rightarrow \infty$ we write instead of $\Lambda, \Lambda^{\prime}=\Pi_{j=1}^{q}\left[1-\left(u_{1}-e_{0}\right) /\left(\theta_{j}-e_{0}\right)\right]$. We can deduce two important relations amongst the $\theta_{j}$. We use the notation $S_{0}=\sum_{f=1}^{q}\left(\theta_{f}-e_{0}\right)^{-1}, S_{1}=\sum_{f=1}^{q}\left(\theta_{f}-e_{1}\right)^{-1}$ and obtain

$$
\begin{gathered}
2(m+1) S_{0}+S_{1}=0 \\
2(m+1) e_{0} S_{0}+e_{1} S_{1}+\frac{1}{2}(\ell-m)(\ell+m+1)=0
\end{gathered}
$$

where we have used the fact that $q=(\ell-m) / 2$. Indeed, the first relation follows by summing equations (72) on $j$, and the second follows by multiplying each equation by $\theta_{j}$ and then summing on $j$. As $\ell \rightarrow \infty$ we see that these relations imply

$$
\hat{S}_{0}=\lim _{\ell \rightarrow \infty} \sum_{f=1}^{\ell} \frac{1}{\theta_{f}-e_{0}}=\frac{\chi^{2}}{4(m+1)}, \quad \hat{S}_{1}=\lim _{\ell \rightarrow \infty} \sum_{f=1}^{\ell} \frac{1}{\theta_{f}-e_{1}}=-\frac{1}{2} \chi^{2} .
$$

(Note that the $\theta_{f}$ themselves depend on $\ell$.) Using the identities satisfied by the zeros of the polynomials $\Lambda^{\prime}$ we can further deduce that

$$
2(m+1) \sum_{f=1}^{q} \frac{1}{\left(\theta_{f}-e_{0}\right)^{2}}+\frac{1}{e_{1}-e_{0}}\left(S_{1}-S_{0}\right)-2 \sum_{g \neq f} \frac{1}{\left(\theta_{g}-e_{0}\right)\left(\theta_{f}-e_{0}\right)}=0,
$$

in addition to the identity

$$
S_{0}^{2}=\sum_{f=1}^{q} \frac{1}{\left(\theta_{f}-e_{0}\right)^{2}}+\sum_{g \neq f} \frac{1}{\left(\theta_{g}-e_{0}\right)\left(\theta_{f}-e_{0}\right)} .
$$

From these two requirements we conclude that as $\ell \rightarrow \infty$

$$
\sum_{g \neq f} \frac{1}{\left(\theta_{g}-e_{0}\right)\left(\theta_{f}-e_{0}\right)} \rightarrow \frac{\chi^{4}}{16(m+1)(m+2)}
$$

Consequently if we evaluate the successive equations in this way we obtain, formally,

$$
\Phi=\left(u_{1}-e_{0}\right)^{\frac{m}{2}}\left(1-\frac{\chi^{2}}{4(m+1)}\left(u_{1}-e_{0}\right)+\frac{\chi^{4}}{32(m+1)(m+2)}\left(u_{1}-e_{0}\right)^{2}-\cdots\right)
$$


which can be recognised [4] as the first few terms of the expansion of

$$
2^{m} \Gamma(m+1) J_{m}\left(\chi \sqrt{u_{1}-e_{0}}\right) .
$$

Note that for a polynomial separated solution of order $q$, with zeros $\theta_{f}$ we have expansions about $e_{s}, s=0,1$, given by

$$
\Lambda\left(u_{i}\right)=\prod_{f=1}^{q}\left(1-\frac{u_{i}-e_{s}}{\theta_{f}-e_{s}}\right)=\sum_{j=1}^{q} c_{j}\left(u_{i}-e_{s}\right)^{j}
$$

where

$$
c_{0}=1, \quad c_{j}=\frac{(-1)^{j}}{j !} \sum_{i_{1}, i_{2}, \cdots, i_{j} \neq} \frac{1}{\left(\theta_{i_{1}}-e_{s}\right) \cdots\left(\theta_{i_{j}}-e_{s}\right)}, \quad j=1,2, \cdots, q .
$$

As $q \rightarrow \infty$ we pass through a family of polynomials and obtain $C_{j}=$ $\lim _{q \rightarrow \infty} c_{j}(q)$, where $C_{j}$ is the coefficient of $\left(u-e_{s}\right)^{j}$ in the separated (nonpolynomial) solution corresponding to the contracted coordinates. Thus these coefficients can be evaluated in terms of limits of the sums (74) of terms involving the zeros of the polynomial solutions.

Let $n_{1}, n_{2}, \cdots, n_{k}$ be a partition of $n$, i.e.,

$$
n_{1} \geq n_{2} \geq \cdots \geq n_{k}>0, \quad n_{1}+\cdots+n_{k}=n .
$$

One can also denote this partition by $1^{t_{1}} 2^{t_{2}} \cdots n^{t_{n}}$ where $t_{1}+\cdots+t_{n}=k$ and $t_{i}$ is the number of occurrences of the integer $i$ in the partition of $n$. We introduce the notation

$$
\left\{n_{1}, \cdots, n_{k}\right\}\left(e_{s}\right) \equiv\left\{1^{t_{i}} \cdots n^{t_{n}}\right\} \equiv \boldsymbol{n}=\sum_{i_{1}, \cdots, i_{k} \neq} \frac{1}{\left(\theta_{i_{1}}-e_{s}\right)^{n_{1}} \cdots\left(\theta_{i_{k}}-e_{s}\right)^{n_{k}}} .
$$

Here the indices $i_{\ell}$ in a given term take values from 1 to $q$, except that the indices in a term are pairwise distinct. The number of terms in the sum is $q ! /(q-k)$ !. We shall show that all such sums, hence their limits, can be evaluated directly from (72).

First we note that the sums multiply in a simple fashion:

$$
\left\{n_{1}, \cdots, n_{k}\right\} \cdot\left\{m_{1}, \cdots, m_{\ell}\right\} \equiv \boldsymbol{n} \cdot \boldsymbol{m}=\sum_{p_{1}, \cdots, p_{k+\ell}} a_{p_{1}, \cdots p_{k+\ell}}\left\{p_{1}, \cdots, p_{k+\ell}\right\}
$$


where the $a$ are integers $\geq 0$, and $p_{1}, \cdots, p_{k+\ell}$ is a partion of $n+m$. (For convenience, we adopt the convention $\left\{n_{1}, \cdots, n_{k}, 0\right\} \equiv\left\{n_{1}, \cdots, n_{k}\right\}$.)

The following are the rules to find the nonzero terms on the right-hand side of (76):

1. Pick any $h$-element subset $n_{s_{1}}, \cdots, n_{s_{h}}\left(s_{1}<s_{2}<\cdots<s_{h}\right)$ of $\boldsymbol{n}$ and any $h$-element subset $m_{r_{1}}, \cdots m_{r_{h}}$ (in any order) of $\boldsymbol{m}$. Then the sum

$$
\left\{n_{s_{1}}+m_{r_{1}}, n_{s_{2}}+m_{r_{2}}, \cdots, n_{s_{h}}+m_{r_{h}}, \boldsymbol{n}^{\prime}, \boldsymbol{m}^{\prime}\right\}
$$

with terms properly reordered, will be included once on the right-hand side of (76). Here $\boldsymbol{n}^{\prime}$ is $\boldsymbol{n}$ with $n_{s_{1}}, \cdots, n_{s_{h}}$ deleted and $\boldsymbol{m}^{\prime}$ is $\boldsymbol{m}$ with $m_{r_{1}}, \cdots m_{r_{h}}$ deleted. This is a partition of $n+m$ which has $h+(n-$ $h)+(m-h)=n+m-h$ nonzero terms.

2. Repeat the preceding step for all $h$-element subsets of $\boldsymbol{n}$ and $\boldsymbol{m}$, and for all $h=0,1, \cdots, \min (k, \ell)$. The sum of all partitions of $n+m$ so constructed is the right-hand side of (76).

Some examples are (all sums depend on a common $e_{s}$ ):

$$
\begin{gathered}
\{1,1\} \cdot\{1,1,1\} \equiv\left\{1^{2}\right\} \cdot\left\{1^{3}\right\}=6\left\{2^{2}, 1\right\}+6\left\{2,1^{3}\right\}+\left\{1^{5}\right\} \\
\{2\} \cdot\left\{1^{2}\right\}=2\{3,1\}+\left\{2,1^{2}\right\}, \quad\{2\} \cdot\{3\}=\{5\}+\{3,2\} \\
\{2,1\} \cdot\left\{1^{2}\right\}=2\{3,2\}+2\left\{3,1^{2}\right\}+2\left\{2^{2}, 1\right\}+\left\{2,1^{3}\right\} .
\end{gathered}
$$

We show how, in principle, one can compute all the sums $\boldsymbol{n}\left(e_{s}\right)$. To be definite we take $s=0$, but a slight modification of the argument works for $s=1$. First, multiplying each term of $(72)$ by $\left(\theta_{j}-e_{0}\right)^{-n+1}$, summing on $j$ and expanding in partial fractions, we obtain the identity:

$$
\begin{aligned}
2(m+1)\{n\}\left(e_{0}\right)-\sum_{j=1}^{n-1} \frac{\{n-j\}\left(e_{0}\right)}{\left(e_{1}-e_{0}\right)^{j}} & +\frac{\{1\}\left(e_{1}\right)}{\left(e_{1}-e_{0}\right)^{n-1}} \\
-4\left(\sum_{\ell=1}^{\left[\frac{n}{2}\right]}\{n-\ell, \ell\}\left(e_{0}\right)-\frac{1}{2}\left\{\frac{n}{2}, \frac{n}{2}\right\}\left(e_{0}\right)\right) & =0,
\end{aligned}
$$

where $\left\{\frac{n}{2}, \frac{n}{2}\right\}\left(e_{0}\right)=0$ if $n$ is odd. We are given $\{1\}\left(e_{0}\right)=S_{0}$ and $\{1\}\left(e_{1}\right)=S_{1}$. It follows from (77), the relation

$$
\{n-k\} \cdot\{k\}=\{n\}+\{n-k, k\}
$$


and a simple induction argument that we can compute all sums $\left\{n_{1}\right\}$ and $\left\{n_{1}, n_{2}\right\}$. Now suppose we know $\left\{n_{1}, \cdots, n_{k}\right\}$ for all $n_{1} \geq \cdots \geq n_{k}>0$ and $k=1,2, \cdots, k_{0}-1$. Then we can use the fact that

$$
\left\{n_{1}, \cdots, n_{k}\right\} \cdot\left\{n_{k+1}\right\}=\left\{n_{1}, \cdots, n_{k+1}\right\}+\text { partitions with } \leq k \text { nonzero terms }
$$

to compute all $\left\{n_{1}, \cdots, n_{k_{0}}\right\}$ such that $n_{1} \geq n_{2} \geq \cdots \geq n_{k_{0}}>0$. Thus we can compute all $\left\{n_{1}, \cdots, n_{k}\right\}$.

We now perform the analogous limiting process for the case of ellipsoidal coordinates on the sphere. We will initially treat these coordinates on the $N$-sphere, and then specialize to the two-sphere to take the limit. These coordinates have the form (6). We want to compute polynomial separable solutions of order $q$ of the Laplace-Beltrami eigenvalue equation and then let $q \rightarrow \infty$. A key observation is the identity (5). Based on this, we look for solutions of the form

$$
\Psi=\Pi_{j=1}^{q}\left(\sum_{k=0}^{N} \frac{X_{k}^{2}}{\theta_{j}-e_{k-1}}\right) \approx \Pi_{j=1}^{q} \Pi_{i}^{N}\left(u_{i}-\theta_{j}\right) .
$$

If we drop the constraint that $\sum_{k} X_{k}^{2}=1$ then the polynomials (79) are homogeneous of order $2 q$ in the coordinates $X_{k}$. By passing to polar coordinates, it is easy to show that the unconstrained function (79) is harmonic, i.e., it satisfies the Euclidean space Laplace equation [10]

$$
\Delta_{N+1} \Psi=0, \quad \Delta_{N+1}=\sum_{h=0}^{N} \partial_{X_{h}}^{2},
$$

if and only if the function (79), constrained to the $N$-sphere satisfies the eigenvalue equation

$$
\hat{\Delta}_{N} \Psi=-2 q(2 q+N-1) \Psi,
$$

where $\hat{\Delta}_{N}$ is the Laplace-Beltrami operator (10) on the $N$-sphere. Substituting the polynomial (79) into (80), we see that this equation is satisfied if and only if $[10,11]$

$$
\sum_{s=0}^{N} \frac{1}{\theta_{j}-e_{s}}+\sum_{k \neq j} \frac{4}{\theta_{j}-\theta_{k}}=0
$$

where $j, k$ take values from 1 to $q$. Moreover, substituting the second equation of (79) into the separation equations (14) we see that the separation constants 
and the zeros of the polynomials are related by

$$
\{1\}\left(e_{s}\right)=\frac{1}{2} \frac{\sum_{\ell=1}^{N} \lambda_{\ell} e_{s}^{N-\ell}}{\Pi_{h \neq s}\left(e_{h}-e_{s}\right)}
$$

where $\lambda_{1}=2 q(2 q+N-1)$.

By computing the inverse matrix to the Vandermonde matrix [12] (page $36)$, we find the identity $(i, t=0, \cdots, N)$

$$
\delta_{i t}=(-1)^{t+N} \sum_{s=0}^{N} \frac{e_{s}^{t}}{\Pi_{\ell \neq s}\left(e_{s}-e_{\ell}\right)} \sum_{i_{1}, \cdots, i_{p} \neq ; i_{1}, \cdots, i_{p} \neq s}^{p=N-i} e_{i_{1}} \cdots e_{i_{p}} .
$$

This allows us to invert relations (83):

$$
\begin{aligned}
\lambda_{\ell} & =2(-1)^{N-\ell+1} \sum_{s=0}^{N}\{1\}\left(e_{s}\right) \sum_{i_{1}, \cdots, i_{\ell} \neq ; i_{1}, \cdots, i_{\ell} \neq s} e_{i_{1}} \cdots e_{i_{\ell}}, \quad \ell=1, \cdots, N \\
0 & =\sum_{s=0}^{N}\{1\}\left(e_{s}\right) .
\end{aligned}
$$

Exactly as in our preceding example, we can define sums $\left\{n_{1}, \cdots, n_{k}\right\}\left(e_{s}\right)$ and a minor alteration of the argument given there shows that all such sums can be evaluated explicitly from (82) and (83). Moreover, if $e_{N} \rightarrow \infty$ according to the prescription following (40), the limits of all these sums exist and are finite.

Now we return to the case $N=2$. See also [5]. On the two-sphere the separation equations have the form $[13,14]$

$$
\begin{gathered}
{\left[-4\left(u_{i}-e_{0}\right)\left(u_{i}-e_{1}\right)\left(u_{i}-e_{2}\right)\left[\partial_{u_{i}}^{2}+\frac{1}{2}\left(\frac{1}{u_{i}-e_{0}}+\frac{1}{u_{i}-e_{1}}+\frac{1}{u_{i}-e_{2}}\right) \partial_{u_{i}}\right]\right.} \\
\left.+\ell(\ell+1) u_{i}+\lambda_{2}\right] \psi_{i}\left(u_{i}\right)=0
\end{gathered}
$$

where $i=1,2$ and $\Psi=\psi_{1}\left(u_{1}\right) \psi_{2}\left(u_{2}\right)$. If we now proceed to the limit $e_{2} \approx$ $\ell^{2} / \chi^{2}$ as $\ell \rightarrow \infty$ with the additional provision that $\lambda_{2} \approx-\tau^{2} \ell^{2} / \chi^{2}$, the separation equations become

$$
\begin{aligned}
{\left[4\left(u_{i}-e_{0}\right)\left(u_{i}-e_{1}\right)\left[\partial_{u_{i}}^{2}+\frac{1}{2}\left(\frac{1}{u_{i}-e_{0}}+\frac{1}{u_{i}-e_{1}}\right) \partial_{u_{i}}\right]\right.} & \\
\left.+\chi^{2} u_{i}-\tau^{2}\right] \varphi_{i}\left(u_{i}\right) & =0 .
\end{aligned}
$$


From previous work we know that in the case of the sphere the corresponding separable solutions are Lamé polynomials and in the case of Euclidean space, Mathieu functions $[4,15]$. We now examine the contraction process as outlined for these coordinate systems. We have

$$
\begin{aligned}
\ell(\ell+1) & =2\left[\left(e_{1}+e_{2}\right) S_{0}+\left(e_{0}+e_{2}\right) S_{1}+\left(e_{0}+e_{1}\right) S_{2}\right] \\
\lambda_{2} & =-2\left[e_{1} e_{2} S_{0}+e_{0} e_{2} S_{1}+e_{0} e_{1} S_{2}\right] \\
0 & =S_{0}+S_{1}+S_{2}
\end{aligned}
$$

where $S_{i}=\sum_{j=1}^{q} \frac{1}{\theta_{j}-e_{i}}$. As $\ell \rightarrow \infty$ these three relations imply

$$
\hat{S}_{2}=-\frac{\chi^{2}}{2}, \quad \hat{S}_{0}+\hat{S}_{1}=\frac{\chi^{2}}{2} \quad e_{1} \hat{S}_{0}+e_{0} \hat{S}_{1}=\frac{\tau^{2}}{2} .
$$

Thus

$$
\hat{S}_{0}=\frac{1}{e_{0}-e_{1}}\left(\frac{e_{0} \chi^{2}}{2}-\frac{\tau^{2}}{2}\right), \quad \hat{S}_{1}=\frac{1}{e_{1}-e_{0}}\left(\frac{e_{1} \chi^{2}}{2}-\frac{\tau^{2}}{2}\right) .
$$

If we now look at the limiting behavior of the polynomials $\Lambda^{\prime}=\Pi_{j=1}^{q}(1-$ $\left.u_{1} / \theta_{j}\right)$ as $\ell \rightarrow \infty$, just as we did previously, we have formally, taking $e_{0}=0$ that

$$
\Lambda^{\prime}=1-\frac{\tau^{2}}{2 e_{1}} u_{1}+\frac{1}{12 e_{1}^{2}}\left[\tau^{2}\left(\tau^{2}+2\right)+2 \chi^{2} e_{1}\right] u_{1}^{2}+\cdots
$$

Note that (87) is obtained by assuming that the limit of an unbounded sum of monomials is equal to the infinite sum of the limits of the individual monomials, and this is by no means obvious. We will give a rigorous proof at the end of this section.

In order to identify this solution we note that with the choice of variables $e_{1}=1, u=\sin ^{2} \theta, q=\chi / 2$ and $a=\chi^{2} / 2-\tau^{2}$ the separation equation for $u$ takes the form $\left(\partial_{\theta}^{2}+a-2 q \cos 2 \theta\right) \varphi=0$ which is the standard form of Mathieu's equation. With appropriate choice of $\tau$ the series developed above for $\Lambda^{\prime}$ will be one of the following (where we use the notation of McLachlan $[16])$.

[1] $\{0,0,0\}$

$$
\Pi_{j=1}^{2 m}\left(1-\frac{u}{\theta_{j}}\right) \rightarrow c e_{2 m}(u, \chi)=\frac{c e_{2 m}(\theta, q)}{c e_{2 m}(0, q)}, \quad a=a_{2 m}
$$


$[2]\{1,0,0\}$

$$
u^{1 / 2} \Pi_{j=1}^{2 m+1}\left(1-\frac{u}{\theta_{j}}\right) \rightarrow s e_{2 m+1}(u, \chi)=\frac{s e_{2 m+1}(\theta, q)}{s e_{2 m+1}^{\prime}(0, q)}, \quad a=b_{2 m+1},
$$

$[3]\{0,1,0\}$

$$
(1-u)^{1 / 2} \Pi_{j=1}^{2 m+1}\left(1-\frac{u}{\theta_{j}}\right) \rightarrow c e_{2 m+1}(u, \chi)=\frac{c e_{2 m+1}(\theta, q)}{c e_{2 m+1}(0, q)}, \quad a=a_{2 m+1},
$$

$[4]\{1,1,0\}$

$$
u^{1 / 2}(1-u)^{1 / 2} \Pi_{j=1}^{2 m+2}\left(1-\frac{u}{\theta_{j}}\right) \rightarrow s e_{2 m+2}(u, \chi)=\frac{s e_{2 m+2}(\theta, q)}{s e_{2 m+2}(0, q)}, a=b_{2 m+2} .
$$

These are the only cases that we need consider as we can readily see that $\left\{\epsilon_{1}, \epsilon_{2}, 0\right\} \approx\left(1 / e_{3}^{1 / 2}\right)\left\{\epsilon_{1}, \epsilon_{2}, \epsilon_{3}\right\}$ as $e_{3} \rightarrow \infty$.

The formal limit (87) needs rigorous justification. We sketch some of the details involved in recovering a periodic Mathieu function from the limits of the Lamé polynomials $\Lambda_{q}\left(u_{1}\right)$. The operator $e_{2}^{-1} I_{2}^{2}$, used in the limiting procedure to calculate $I_{2}^{2}$, takes the form

$$
e_{2}^{-1} I_{2}^{2}=L_{01}^{2}+\frac{e_{1}}{e_{2}} L_{02}^{2}+\frac{e_{0}}{e_{2}} L_{12}^{2},
$$

and from the well known action of the operators $L_{01}, L_{02}, L_{12}$ defining the $2 \ell+1$ dimensional irreducible representation $D_{\ell}$ of $S O(3)$, [2], and Gers̆gorin's theorem, [12], we can verify that as $\ell$ grows without bound, each of the eigenvalues $e_{2}^{-1} \lambda_{2}$ of $e_{2}^{-1} I_{2}^{2}$ lies in one of the intervals

$$
\left|\frac{\lambda_{2}}{e_{2}}+4 k^{2}+\frac{\chi^{2}}{2}\left(e_{0}+e_{1}\right)\right| \leq\left(e_{1}-e_{0}\right) \chi^{2}, \quad k=0,1, \cdots .
$$

Further, for $k$ and $\ell$ suitably large so that the $k$-interval doesn't overlap any of the other intervals, there is exactly one eigenvalue $\lambda_{2}$ in the $k$-interval for fixed $k, \ell$. Since this interval is compact, there is a sequence of eigenvalues $\lambda_{2, q} \chi^{2} / \ell^{2}, \ell=2 q$, such that $\lambda_{2, q} \chi^{2} / \ell^{2} \rightarrow-\tau^{2}$ as $q \rightarrow \infty$, with $-\tau^{2}$ in the $k$-interval. We will show that $-\tau^{2}$ is a discrete eigenvalue corresponding to the Mathieu equation. 
With a very similar argument to that in (75)-(78) we can determine all of the sums (75). In particular, these all have finite limits for $e_{2}=\ell^{2} / \chi^{2}$ as $\ell=2 q \rightarrow \infty$. Since the sums $\{2\}_{q}\left(e_{s}\right)$ are finite and have finite limits as $q \rightarrow \infty$ for $s=0,1,2$, it follows that there exists a positive constant $\kappa$ such that $\left|\theta_{p}(q)-e_{s}\right|^{-1}<\kappa$ for $s=0,1, p=1,2, \cdots q$ and all $q$. Since $\{2\}_{q}\left(e_{0}\right)$ is uniformly bounded in $q$, there must be a strict upper bound on the number of zeros $\theta_{p}(q)$ in the interval $e_{0}<\theta_{p}(q)<e_{1}$, uniform for all $q$. Call this upper bound $P$.

Each polynomial in the sequence $\Lambda_{q}\left(u_{1}\right), q=0,1, \cdots$ can be written in the form

$$
\Lambda_{q}\left(u_{1}\right)=\prod_{p=1}^{P_{q}}\left(1-\frac{u_{1}-e_{0}}{\theta_{p}(q)-e_{0}}\right) \prod_{p=P_{q}+1}^{q}\left(1-\frac{u_{1}-e_{0}}{\theta_{p}(q)-e_{0}}\right)=\Lambda_{P_{q}}^{(1)} \Lambda_{q-P_{q}}^{(2)},
$$

where

$$
\begin{gathered}
e_{0}<\theta_{p}(q)<e_{1}, \quad p=1, \cdots, P_{q}, \\
e_{1}<\theta_{p}(q)<e_{2}(q), \quad p=P_{q}+1, \cdots, q,
\end{gathered}
$$

and $P_{q} \leq P$. Note that the polynomial $\Lambda^{(2)}\left(u_{1}\right)$ takes the value 1 for $u_{1}=e_{0}$ and that this polynomial is strictly positive and monotone decreasing in the interval $e_{0} \leq u_{1} \leq e_{1}$. It follows that the polynomials $\left\{\Lambda_{q}\left(u_{1}\right)\right\}$ are uniformly bounded on $\left[e_{0}, e_{1}\right]$ for all $q$. Furthermore, since $\{j\}_{q}\left(e_{0}\right),\{j\}_{q}\left(e_{1}\right)$ are uniformly bounded for $j=1,2$ it follows easily that the derivatives $\frac{d^{k}}{d u_{1}{ }^{k}} \Lambda_{q}\left(u_{1}\right)$, $k=1,2,3$ are also uniformly bounded in absolute value. Thus each of the families $\left\{\Lambda_{q}\right\},\left\{\Lambda_{q}^{\prime}\right\},\left\{\Lambda_{q}^{\prime \prime}\right\}$ is equicontinuous and uniformly bounded. Using Arzelà's Theorem, [17], we can choose a subsequence $\left\{\lambda_{q^{\prime}}\right\}$ of $\left\{\Lambda_{q}\right\}$ such that

$$
\Lambda_{q^{\prime}}\left(u_{1}\right) \rightarrow \varphi\left(u_{1}\right), \quad \Lambda_{q^{\prime}}^{\prime}\left(u_{1}\right) \rightarrow \varphi^{\prime}\left(u_{1}\right), \quad \Lambda_{q^{\prime}}^{\prime \prime}\left(u_{1}\right) \rightarrow \varphi^{\prime \prime}\left(u_{1}\right),
$$

uniformly on $\left[e_{0}, e_{1}\right]$ as $q^{\prime} \rightarrow \infty$. Hence, $\varphi$ satisfies (85) and, if $e_{0}=0$, the expansion (87). Since $\varphi$ is bounded on $\left[e_{0}, e_{1}\right]$ it is a periodic Mathieu function and $-\tau^{2}$ is in the point spectrum.

\section{The complex two-sphere and plane}

A main thrust of this paper is to demonstrate how the notion of contraction based on the classical examples extends to the various separable coordinate 
systems on the two dimensional complex sphere and two dimensional complex euclidean space. The easiest way to see this is to use the algebraic form of the various coordinate systems. We list here the separable coordinates, first on the complex sphere. We do this in algebraic form and for a sphere of radius $R$. Since the coordinates are complex, there are now separable coordinate systems permitted in addition to the real systems listed in $\S 3,[6,18,19]$. In particular, systems $\{2\},\{4\},\{5\},(2)$ and (5) are new. Moreover, the parameters $e_{j}$ are now complex numbers so the previous ordering of the $e_{j}$ no longer is appropriate.

\section{THE TWO-SPHERE}

\section{$\{1\}$ Spherical coordinates}

$$
s_{1}^{2}=R^{2} \frac{\left(u_{1}-e_{0}\right)\left(u_{2}-a_{0}\right)}{\left(e_{1}-e_{0}\right)\left(a_{1}-a_{0}\right)}, \quad s_{2}^{2}=R^{2} \frac{\left(u_{1}-e_{0}\right)\left(u_{2}-a_{1}\right)}{\left(e_{1}-e_{0}\right)\left(a_{0}-a_{1}\right)}, \quad s_{3}^{2}=R^{2} \frac{\left(u_{1}-e_{1}\right)}{\left(e_{0}-e_{1}\right)}
$$

The infinitesimal distance is given by

$$
d s^{2}=R^{2}\left[-\frac{d u_{1}^{2}}{4\left(u_{1}-e_{0}\right)\left(u_{1}-e_{1}\right)}+\frac{\left(u_{1}-e_{0}\right)}{4\left(e_{0}-e_{1}\right)} \frac{d u_{2}^{2}}{\left(u_{2}-a_{0}\right)\left(u_{2}-a_{1}\right)}\right]
$$

and the diagram is

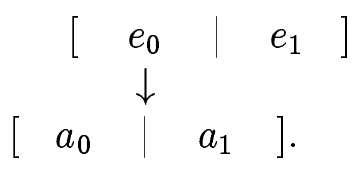

$\{2\}$ Horospherical coordinates

$$
\begin{gathered}
\left(s_{1}+i s_{2}\right)^{2}=R^{2} \frac{\left(u_{1}-e_{0}\right)\left(u_{2}-a_{0}\right)}{\left(e_{1}-e_{0}\right)}, \quad\left(s_{1}^{2}+s_{2}^{2}\right)=R^{2} \frac{\left(u_{1}-e_{0}\right)}{\left(e_{1}-e_{0}\right)}, \\
s_{3}^{2}=R^{2} \frac{\left(u_{1}-e_{1}\right)}{\left(e_{0}-e_{1}\right)}
\end{gathered}
$$

where the infinitesmal distance and diagram are given by

$$
d s^{2}=\frac{R^{2}}{2}\left[-\frac{d u_{1}^{2}}{\left(u_{1}-e_{1}\right)\left(u_{1}-e_{2}\right)}+\frac{\left(u_{1}-e_{1}\right)}{\left(e_{1}-e_{2}\right)} \frac{d u_{2}^{2}}{\left(u_{2}-a_{0}\right)^{2}}\right],
$$


\{3\} Elliptical coordinates

$$
\begin{aligned}
& {\left[\begin{array}{c|ll}
e_{0} & \mid e_{1} & \\
\downarrow &
\end{array}\right.} \\
& {\left[\begin{array}{l}
a_{0}^{2}
\end{array}\right] .}
\end{aligned}
$$

$$
\begin{gathered}
s_{1}^{2}=R^{2} \frac{\left(u_{1}-e_{0}\right)\left(u_{2}-e_{0}\right)}{\left(e_{0}-e_{1}\right)\left(e_{0}-e_{2}\right)}, \quad s_{2}^{2}=R^{2} \frac{\left(u_{1}-e_{1}\right)\left(u_{2}-e_{1}\right)}{\left(e_{1}-e_{0}\right)\left(e_{1}-e_{2}\right)}, \\
s_{3}^{2}=R^{2} \frac{\left(u_{1}-e_{2}\right)\left(u_{2}-e_{2}\right)}{\left(e_{2}-e_{1}\right)\left(e_{2}-e_{0}\right)}
\end{gathered}
$$

The infinitesimal distance and diagram are given by

$$
\begin{gathered}
d s^{2}=-\frac{1}{4} R^{2}\left(u_{1}-u_{2}\right)\left[\frac{d u_{1}^{2}}{\left(u_{1}-e_{0}\right)\left(u_{1}-e_{1}\right)\left(u_{1}-e_{2}\right)}-\frac{d u_{2}^{2}}{\left(u_{2}-e_{0}\right)\left(u_{2}-e_{1}\right)\left(u_{2}-e_{2}\right)}\right], \\
{\left[e_{0}\left|e_{1}\right| e_{2}\right] .}
\end{gathered}
$$

\{4\} Degenerate elliptical coordinates of type 1

$$
\begin{gathered}
(x+i y)^{2}=R^{2} \frac{\left(u_{1}-e_{0}\right)\left(u_{2}-e_{0}\right)}{\left(e_{1}-e_{0}\right)}, \quad\left(x^{2}+y^{2}\right)=-R^{2} \frac{\partial}{\partial e_{0}}\left[\frac{\left(u_{1}-e_{0}\right)\left(u_{2}-e_{0}\right)}{\left(e_{1}-e_{0}\right)}\right], \\
z^{2}=R^{2} \frac{\left(u_{1}-e_{1}\right)\left(u_{2}-e_{1}\right)}{\left(e_{0}-e_{1}\right)^{2}}
\end{gathered}
$$

The infinitesimal distance and diagram are given by

$d s^{2}=-\frac{1}{4} R^{2}\left(u_{1}-u_{2}\right)\left[\frac{d u_{1}^{2}}{\left(u_{1}-e_{0}\right)^{2}\left(u_{1}-e_{1}\right)}-\frac{d u_{1}^{2}}{\left(u_{1}-e_{0}\right)^{2}\left(u_{1}-e_{1}\right)}\right], \quad\left[e_{0}^{2} \mid e_{1}\right]$.

$\{5\}$ Degenerate elliptic coordinates of type 2

$$
\begin{gathered}
(x+i y)^{2}=R^{2}\left(u_{1}-e_{0}\right)\left(u_{2}-e_{0}\right), \quad 2(x+i y) z=-R^{2} \frac{\partial}{\partial e_{0}}\left[\left(u_{1}-e_{0}\right)\left(u_{2}-e_{0}\right)\right], \\
x^{2}+y^{2}+z^{2}=R^{2}
\end{gathered}
$$

The infinitesimal distance and diagram are given by

$$
d s^{2}=-\frac{1}{4} R^{2}\left(u_{1}-u_{2}\right)\left[\frac{d u_{1}^{2}}{\left(u_{1}-e_{0}\right)^{3}}-\frac{d u_{2}^{2}}{\left(u_{2}-e_{0}\right)^{3}}\right], \quad\left[e_{0}^{3}\right] .
$$


For Euclidean two-space we have the following coordinate systems:

\section{EUCLIDEAN TWO-SPACE}

\section{(1) Ellipsoidal coordinates}

$$
x^{2}=c^{2} \frac{\left(v_{1}-b_{0}\right)\left(v_{2}-b_{0}\right)}{\left(b_{0}-b_{1}\right)}, \quad y^{2}=c^{2} \frac{\left(v_{1}-b_{1}\right)\left(v_{2}-b_{1}\right)}{\left(b_{1}-b_{0}\right)},
$$

with infinitesimal distance given by

$$
d s^{2}=-\frac{c^{2}}{4}\left(v_{1}-v_{2}\right)\left[\frac{d v_{1}^{2}}{\left(v_{1}-b_{0}\right)\left(v_{1}-b_{1}\right)}-\frac{d v_{2}^{2}}{\left(v_{2}-b_{0}\right)\left(v_{2}-b_{1}\right)}\right],
$$

and diagram $<b_{0} \mid b_{1}>$.

(2) Degenerate elliptic coordinates

$$
(x+i y)^{2}=c^{2}\left(v_{1}-e_{0}\right)\left(v_{2}-e_{0}\right), \quad x^{2}+y^{2}=c^{2}\left(2 e_{0}-v_{1}-v_{2}\right),
$$

with infinitesimal distance given by

$$
d s^{2}=-\frac{c^{2}}{4}\left(v_{1}-v_{2}\right)\left[\frac{d v_{1}^{2}}{\left(v_{1}-e_{0}\right)^{2}}-\frac{d v_{2}^{2}}{\left(v_{2}-e_{0}\right)^{2}}\right],
$$

and diagram $<e_{0}^{2}>$.

(3) Spherical coordinates

$$
x^{2}=\left(v_{1}-e_{0}\right) \frac{\left(v_{2}-a_{0}\right)}{\left(a_{1}-a_{0}\right)}, \quad y^{2}=\left(v_{1}-e_{0}\right) \frac{\left(v_{2}-a_{1}\right)}{\left(a_{0}-a_{1}\right)},
$$

with infinitesimal distance and diagram given by

$$
\begin{gathered}
d s^{2}=\frac{1}{4}\left[\frac{d v_{1}^{2}}{\left(v_{1}-e_{0}\right)}-\left(v_{1}-e_{0}\right) \frac{d v_{2}^{2}}{\left(v_{2}-a_{0}\right)\left(v_{2}-a_{1}\right)}\right], \\
\left\langle\begin{array}{cccc}
e_{0} & \mid & e_{1}
\end{array}\right\rangle \\
{\left[\begin{array}{llll}
\downarrow & a_{0} & \mid & a_{1}
\end{array}\right] .}
\end{gathered}
$$

(4) Parabolic coordinates 


$$
x^{2}=-c^{2}\left(v_{1}-e_{0}\right)\left(v_{2}-e_{0}\right), \quad y=\frac{c}{2}\left(v_{1}+v_{2}\right)-c e_{0}
$$

with infinitesimal distance and diagram given by

$$
d s^{2}=\frac{c^{2}}{4}\left(v_{1}-v_{2}\right)\left[\frac{d v_{1}^{2}}{\left(v_{1}-e_{0}\right)}-\frac{d v_{2}^{2}}{\left(v_{2}-e_{0}\right)}\right], \quad\left(e_{0}\right) .
$$

(5) Degenerate parabolic coordinates

$$
x=-\frac{c}{8}\left(v_{1}-v_{2}\right)^{2}+\frac{c}{4}\left(v_{1}+v_{2}\right), \quad y=i\left[-\frac{c}{8}\left(v_{1}-v_{2}\right)^{2}-\frac{c}{4}\left(v_{1}+v_{2}\right)\right],
$$

with infinitesimal distance and diagram given by

$$
d s^{2}=\frac{c^{2}}{4}\left(v_{1}-v_{2}\right)\left(d v_{1}^{2}-d v_{2}^{2}\right), \quad\{\phi\}
$$

We work out a few cases. As a first example let us consider degenerate elliptic coordinates of type 1 . The Helmholtz equation for this coordinate system has the form

$$
\begin{gathered}
-\frac{4}{R^{2}\left(u_{1}-u_{2}\right)}\left[\left(u_{1}-e_{0}\right) \sqrt{u_{1}-e_{2}} \partial_{u_{1}}\left(u_{1}-e_{0}\right) \sqrt{u_{1}-e_{1}} \partial_{u_{1}}-\right. \\
\left.\left(u_{2}-e_{0}\right) \sqrt{u_{2}-e_{1}} \partial_{u_{2}}\left(u_{2}-e_{0}\right) \sqrt{u_{2}-e_{1}} \partial_{u_{2}}\right] \Psi=-\frac{\ell(\ell+1)}{R^{2}} \Psi .
\end{gathered}
$$

The separable solutions are $\Psi=\psi_{1}\left(u_{1}\right) \psi_{2}\left(u_{2}\right)$ with separable equations

$$
4\left(u-e_{0}\right) \sqrt{u-e_{1}} \partial_{u}\left(u-e_{0}\right) \sqrt{u-e_{1}} \partial_{u} \psi+(\ell(\ell+1) u+\mu) \psi=0 .
$$

If we choose the new variable $z=\sqrt{\left(u-e_{2}\right) /\left(u-e_{1}\right)}$ then the separation equation has the form

$$
\left[\left(1-z^{2}\right) \partial_{z}^{2}-2 z \partial_{z}+\nu(\nu+1)-\frac{\left(\ell+\frac{1}{2}\right)^{2}}{1-z^{2}}\right] \varphi=0
$$

where

$$
\psi=\left(u-e_{0}\right)^{-1 / 4} \varphi, \quad \nu(\nu+1)=\frac{1}{4}+\frac{\mu-e_{0} \ell(\ell+1)}{e_{0}-e_{1}}
$$


The equation for $\varphi$ is clearly recognisable as having the solutions $\varphi=$ $P_{\nu}^{\ell+\frac{1}{2}}(z)$ and $Q_{\nu}^{\ell+\frac{1}{2}}(z)$, Legendre functions of the first and second kind [4]. From the point of view of contractions we are interested in the limit $e_{1} \rightarrow \infty$. This corresponds to the diagrammatic limit

$$
\lim _{e_{1} \rightarrow \infty}\left[e_{0}^{2} \mid e_{1}\right] \rightarrow<e_{0}^{2}>\text {. }
$$

From the form of the infinitesmal distance we expect to obtain degenerate elliptic coordinates in euclidean space. Indeed if we consider $e_{1} \rightarrow n^{2}, \ell+\frac{1}{2} \rightarrow$ $n \chi$ for the separation functions $\psi$ then one can derive

$$
\begin{gathered}
\Gamma(1-n \chi) P_{\nu}^{n \chi}\left(\sqrt{\frac{u-n^{2}}{u-e_{0}}}\right) \rightarrow-i \sqrt{\frac{\pi \chi}{2}}\left(u-e_{0}\right)^{1 / 4} H_{\nu+\frac{1}{2}}^{(2)}\left(\chi \sqrt{u-e_{0}}\right) \\
\frac{(n \chi)^{\nu+1}}{\Gamma(1+\nu+n \chi)} Q_{\nu}^{n \chi}\left(\sqrt{\frac{u-n^{2}}{u-e_{0}}}\right) \rightarrow \sqrt{\frac{\pi \chi}{2}} e^{\frac{i \pi}{4}}\left(u-e_{0}\right)^{\frac{1}{4}} J_{\nu+\frac{1}{2}}\left(\chi \sqrt{u-e_{0}}\right)
\end{gathered}
$$

as $n \rightarrow \infty$. Here $H_{\rho}^{(2)}(w)$ is a Hankel function of the second kind.

The second possible limit is when $e_{0} \rightarrow \infty$. This corresponds to the diagrammatic limit

$$
\lim _{e_{0} \rightarrow \infty}\left[e_{0}^{2} \mid e_{1}\right] \rightarrow\left(e_{1}\right)
$$

In this case we consider the equation for $\varphi$ and take the limits

$$
e_{0} \rightarrow n, \quad \ell \rightarrow n \alpha, \quad \nu(\nu+1) \rightarrow-n \alpha(1-4 \chi)+n^{2} \alpha^{2}
$$

In particular if we choose $\nu$ such that

$$
\nu \rightarrow-n \alpha-1+2 \chi+\cdots
$$

then we obtain

$$
\begin{gathered}
2^{-n \alpha} \Gamma\left(\frac{1}{4}+\chi-\alpha n\right) P_{-n \alpha-1+2 \chi \cdots(}^{n \alpha+\frac{1}{2}}\left(\sqrt{\frac{u-e_{1}}{u-n}}\right) \rightarrow \sqrt{2 \pi} e^{-\frac{i \pi}{4}} e^{-\frac{1}{2} \alpha\left(u-e_{1}\right)} \times \\
\left\{\frac{1}{\Gamma\left(\frac{3}{4}-\chi\right)}{ }_{1} F_{1}\left(\frac{1}{4}-\chi, \frac{1}{2}, \alpha\left(u-e_{1}\right)\right)-\frac{2 i \alpha^{\frac{1}{2}}\left(u-e_{1}\right)^{\frac{1}{2}}}{\Gamma\left(\frac{1}{4}+\chi\right)}{ }_{1} F_{1}\left(\frac{3}{4}-\chi, \frac{3}{2}, \alpha\left(u-e_{1}\right)\right)\right\} ; \\
2^{-n \alpha} e^{-\frac{3 i \pi}{2}} \Gamma\left(\frac{1}{4}+\chi-n \alpha\right) Q_{-n \alpha-1+2 \chi \cdots}^{n \alpha+\frac{1}{2}}\left(\sqrt{\frac{u-e_{1}}{u-n}}\right) \rightarrow \pi^{\frac{1}{2}} e^{\frac{i \pi}{2}(1-\chi)} e^{-\frac{1}{2} \alpha\left(u-e_{1}\right)} \times
\end{gathered}
$$


$\left\{2^{-\frac{1}{2}} \Gamma\left(\frac{1}{4}+\chi\right){ }_{1} F_{1}\left(\frac{1}{4}-\chi, \frac{1}{2}, \alpha\left(u-e_{1}\right)\right)-i \alpha^{\frac{1}{2}}\left(u-e_{1}\right)^{\frac{1}{2}} 2^{\frac{1}{2}} \Gamma\left(\frac{3}{4}+\chi\right)_{1} F_{1}\left(\frac{3}{4}-\chi, \frac{3}{2}, \alpha\left(u-e_{1}\right)\right\}\right.$, as $n \rightarrow \infty$.

There is one more interesting limit of this type. This corresponds to letting $e_{0} \rightarrow \infty$ in degenerate elliptic coordinates of type 2 , i.e.,

$$
\lim _{e_{0} \rightarrow \infty}\left[e_{0}^{3}\right] \rightarrow\{\phi\}
$$

The Laplace-Beltrami eigenvalue equation in these coordinates is

$$
\begin{gathered}
4\left[\left(u_{1}-e_{0}\right)^{3} \partial_{u_{1}}^{2}+3\left(u_{1}-e_{0}\right)^{2} \partial_{u_{1}}-\left(u_{2}-e_{0}\right)^{3} \partial_{u_{2}}^{2}+3\left(u_{2}-e_{0}\right)^{2} \partial_{u_{2}}\right] \Psi \\
-\ell(\ell+1)\left(u_{1}-u_{2}\right) \Psi=0 .
\end{gathered}
$$

The corresponding separation equations have the form

$$
\left[4\left(u-e_{0}\right)^{3} \partial_{u}^{2}+3\left(u-e_{0}\right)^{2} \partial_{u}-\ell(\ell+1)\left(u-e_{0}\right)+4 \lambda\right] \psi(u)=0 .
$$

The solutions of this equation are readily calculated to be

$$
\psi(u)=\left(u-e_{0}\right)^{-1 / 4} C_{\ell+\frac{1}{2}}\left(2 \sqrt{\frac{\lambda}{r}}\right)
$$

where $C_{\nu}(z)$ is a Bessel function [4]. For $e_{0} \rightarrow \infty$ we would like to end up with degenerate elliptic coordinates. The way to achieve this is to take

$$
e_{0} \rightarrow n^{2}, \quad \ell \rightarrow 2 \chi n^{3}, \quad \lambda \rightarrow-\chi^{2} n^{8}-\frac{1}{2} \chi n^{5}+\mu n^{6}
$$

Then we obtain the limit

$$
\left(2 \chi n^{3}+\frac{1}{2}\right)^{\frac{1}{2}} J_{2 \chi n^{3}+\frac{1}{2}}\left(2 \sqrt{\frac{\chi^{2} n^{8}+\frac{1}{2} \chi n^{5}-\mu n^{6}}{n^{2}-r}}\right) \rightarrow \frac{1}{\pi}\left[\frac{2}{3 \chi}\left(\mu-\chi^{2} r\right)\right]^{\frac{1}{2}} K_{\frac{1}{3}}\left(\frac{\left(\mu-\chi^{2} r\right)^{\frac{3}{2}}}{2^{\frac{1}{2}} \chi^{2}}\right),
$$

the latter function being a solution of the separation equations.

\section{Contractions between coordinate systems within a space}

In the previous section we have examined the relations between the special functions obtained by contracting from the complex two dimensional sphere 
to the complex Euclidean plane. Here we examine the consequences of taking limits of coordinate systems within each of the manifolds $E_{2}$ and $S_{2}$ and derive the relations between the corresponding special functions.

[1] The complex two sphere.

(a) Consider the limit associated with the diagrammatic scheme

$$
\left[e_{0}\left|e_{1}\right| e_{2}\right] \rightarrow\left[e_{0}^{2} \mid e_{1}\right]
$$

or equivalently $e_{2} \rightarrow e_{0}$. In this limiting case the various forms of Lamé polynomials have as their limiting forms suitable Legendre polynomials. For the purposes of this case it is convenient to consider the separation equations in degenerate ellipsoidal coordinates. Indeed if we choose the variable $x=$ $\sqrt{\left(u-e_{2}\right) /\left(e_{1}-e_{2}\right)}$ then the separation equations have the form

$$
\left[\left(1-x^{2}\right) \partial_{x}^{2}-2 x \partial_{x}+\ell(\ell+1)-\frac{m^{2}}{1-x^{2}}\right] \varphi=0
$$

where $m^{2}=\left(\mu+e_{1} \ell(\ell+1)\right) /\left(e_{1}-e_{2}\right)$. The solutions are Legendre functions $P_{\ell}^{m}(x)$ and $Q_{\ell}^{m}(x)$. In order to take the limit we examine the case of a Lamé polynomial of the species which can be written in the form $\Pi_{j=1}^{q}\left(1-u / \theta_{j}\right)$ where the $\theta_{j}$ are the solutions of the Niven equations (82). We write the $\theta_{1}$ equation in the form

$$
\begin{gathered}
{\left[\left(\theta_{1}-e_{1}\right) \quad\left(\theta_{1}-e_{2}\right)+\left(\theta_{1}-e_{1}\right)\left(\theta_{1}-e_{0}\right)+\left(\theta_{1}-e_{0}\right)\left(\theta_{1}-e_{2}\right)\right] \prod_{i=1}^{q}\left(\theta_{1}-\theta_{i}\right)} \\
+4 \quad\left(\theta_{1}-e_{0}\right)\left(\theta_{1}-e_{1}\right)\left(\theta_{1}-e_{2}\right)\left[\left(\theta_{1}-\theta_{3}\right) \ldots\left(\theta_{1}-\theta_{q}\right)+\cdots\right]=0 .
\end{gathered}
$$

We see that the equation is satisfied when $e_{2}=e_{0}$ by the choice $\theta_{1}=e_{0}$. From this observation the limit $e_{2} \rightarrow e_{0}$ can be considered to be accompanied by the reqirement that $\sigma(<q)$ of the $\theta_{j}$ 's take the value $e_{0}$. The remaining $\theta_{j}$ 's then satisfy the equations

$$
\frac{4 \sigma+2}{\theta_{j}-e_{0}}+\frac{1}{\theta_{j}-e_{1}}+\sum_{i \neq j} \frac{4}{\theta_{j}-\theta_{i}}=0
$$

for $i, j=\sigma+1, \cdots, q$. We are at liberty to take $e_{0}=1$ and $e_{1}=0$. The appropriate limit in this case is

$$
\Pi_{j=1}^{\sigma+2 r}\left(1-\frac{u}{\theta_{j}}\right) \rightarrow(-1)^{\sigma+r} 2^{2 r} \frac{(r-\sigma) !(r+\sigma) !}{(2 r+2 \sigma) !} P_{2 r}^{2 \sigma}(\sqrt{u})
$$


for $r \geq \sigma$. Other families of Lamé polynomials have similar limits:

$$
(u-1)^{\frac{1}{2}} \Pi_{j=1}^{\sigma+2 r+1}\left(1-\frac{u}{\theta_{j}}\right) \rightarrow(-1)^{\sigma} 2^{2 r+1} \frac{(r-\sigma) !(r+\sigma+1) !}{(2 r+2 \sigma+2) !} P_{2 r+1}^{2 \sigma+1}(\sqrt{u})
$$

where

$$
\begin{gathered}
\frac{4 \sigma+4}{\theta_{j}-1}+\frac{1}{\theta_{j}}+\sum_{i \neq j} \frac{4}{\theta_{j}-\theta_{i}}=0 \\
u^{\frac{1}{2}} \Pi_{j=1}^{\sigma+2 r+1}\left(1-\frac{u}{\theta_{j}}\right) \rightarrow 2^{2 r+1} \frac{(r-\sigma) !(r+\sigma+1) !}{(2 r+2 \sigma+2) !} P_{2 r+1}^{2 \sigma}(\sqrt{u})
\end{gathered}
$$

where

$$
\frac{4 \sigma+4}{\theta_{j}-1}+\frac{3}{\theta_{j}}+\sum_{i \neq j} \frac{4}{\theta_{j}-\theta_{i}}=0
$$

and

$$
u^{\frac{1}{2}}(u-1)^{\frac{1}{2}} \Pi_{j=1}^{\sigma+2 r}\left(1-\frac{u}{\theta_{j}}\right) \rightarrow(-1)^{\sigma+r+1} 2^{2 r} \frac{(r-\sigma-1) !(r+\sigma+1) !}{(2 r+2 \sigma+2) !} P_{2 r}^{2 \sigma+1}(\sqrt{u})
$$

where

$$
\frac{4 \sigma+2}{\theta_{j}-1}+\frac{3}{\theta_{j}}+\sum_{i \neq j} \frac{4}{\theta_{j}-\theta_{i}}=0 .
$$

In all these cases it is understood that $i, j=\sigma+1, \cdots, q$ where $q=\sigma+2 r$ or $\sigma+2 r+1$ as the case may be. We are of course assuming that $\theta_{1}, \theta_{2}, \ldots, \theta_{\sigma}$ are all equal to 1 .

(b) In this case let us consider the limit $\left[e_{0}^{2} \mid e_{1}\right] \rightarrow\left[e_{0}^{3}\right]$, which is tantamount to $e_{1} \rightarrow e_{0}$. From what we have done previously we know that the special functions associated with the coordinate system $\left[e_{0}^{2} \mid e_{1}\right]$ are Legendre functions $P_{\nu}^{-\ell-\frac{1}{2}}\left(\sqrt{\frac{u-e_{1}}{u-e_{0}}}\right)$ and $Q_{\nu}^{-\ell-\frac{1}{2}}\left(\sqrt{\frac{u-e_{1}}{u-e_{0}}}\right)$ where $\nu(\nu+1)=1 / 4+$ $\left(\mu-e_{0} \ell(\ell+1)\right) /\left(e_{0}-e_{1}\right)$. If we take $e_{1}=e_{0}+\epsilon^{2}$ the limits are

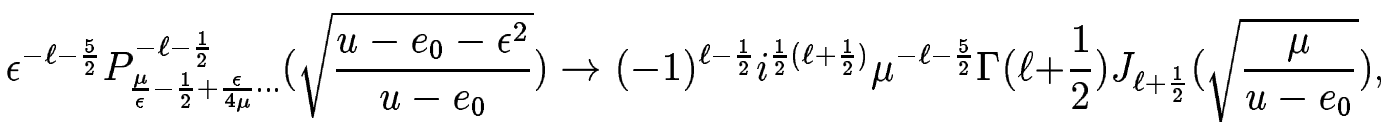

$$
\begin{aligned}
& e^{i \pi\left(\ell+\frac{1}{2}\right)} \epsilon^{-\ell-\frac{5}{2}} Q_{\frac{\mu}{\epsilon}-\frac{1}{2}+\frac{\epsilon}{4 \mu} \cdots}^{-\ell-\frac{1}{2}}\left(\sqrt{\frac{u-e_{0}-\epsilon^{2}}{u-e_{0}}}\right) \rightarrow \\
& \mu^{-\ell-\frac{5}{2}}\left[\frac{1}{2} i^{-\ell-\frac{1}{2}} \Gamma\left(\frac{1}{2}-\ell\right) \Gamma\left(-\frac{1}{2}+\ell\right) J_{-\ell-\frac{1}{2}}\left(\sqrt{\frac{\mu}{u-e_{0}}}\right)\right.
\end{aligned}
$$




$$
\left.+2\left(\frac{3}{2}+\ell\right) \Gamma\left(-\ell-\frac{1}{2}\right)(-1)^{\frac{1}{2}\left(\ell+\frac{1}{2}\right)} J_{\ell+\frac{1}{2}}\left(\sqrt{\frac{\mu}{u-e_{0}}}\right)\right]
$$

[2] Two dimensional Euclidean space.

(a). Let us consider the diagram $<e_{0} \mid e_{1}>\rightarrow<e_{0}^{2}>$, i.e., a transformation from elliptical coordinates in the plane to degenerate elliptical coordinates. In the case of elliptical coordinates the basic separation equations have the form

$4\left(v-e_{0}\right)\left(v-e_{1}\right)\left[\partial_{v}^{2}+\frac{1}{2}\left\{\frac{1}{u-e_{0}}+\frac{1}{u-e_{1}}\right\} \partial_{v}\right] \varphi+\left(-\nu^{2}+\chi^{2} c^{2}\left(v-e_{0}\right)\right) \varphi=0$.

We can assume that $e_{0}=0$ and take $e_{1}=b$. If we set $v=b \cosh ^{2} \theta$ then the separation equation becomes

$$
\left(\partial_{\theta}^{2}-a+2 k^{2} \cosh 2 \theta\right) \Phi=0
$$

which is a recognised form of Mathieu's equation with $k^{2}=\chi^{2} c^{2} b / 4, a=$ $-\nu^{2}-\chi^{2} c^{2} b / 2$. If $b \rightarrow 0$ in the separation equation, the resulting solutions have the form $C_{\nu}\left(\chi c v^{1 / 2}\right)$. In order to be consistent with our limiting procedures we introduce an algebraic notation for Mathieu functions according to

$$
C E_{\nu}(v, q)=C e_{\nu}(\theta, q), \quad S E_{\nu}(v, q)=S e_{\nu}(\theta, q)
$$

where $q=k^{2}=\frac{1}{4} \chi^{2} c^{2} b$ and $v$ and $\theta$ are related as above. The corresponding limits that we seek are then

$$
\begin{gathered}
\frac{(-1)^{2 m} A_{0}^{(2 m)}}{c e_{2 m}(0, q) c e_{2 m}\left(\frac{\pi}{2}, q\right)} C E_{2 m}(v, q) \rightarrow J_{2 m}\left(\chi c v^{1 / 2}\right), \\
\frac{(-1)^{m+1}\left(\frac{1}{2} \chi c b^{1 / 2}\right) A_{1}^{(2 m+1)}}{c e_{2 m+1}(0, q) c e_{2 m+1}^{\prime}\left(\frac{\pi}{2}, q\right)} C E_{2 m+1}(v, q) \rightarrow J_{2 m+1}\left(\chi c v^{1 / 2}\right), \\
\frac{(-1)^{m}\left(\frac{1}{2} \chi c b^{1 / 2}\right) B_{1}^{(2 m+1)}}{s e_{2 m+1}^{\prime}(0, q) s e_{2 m+}\left(\frac{\pi}{2}, q\right)} S E_{2 m+1}(v, q) \rightarrow J_{2 m+1}\left(\chi c v^{1 / 2}\right),
\end{gathered}
$$

and

$$
\frac{(-1)^{m+1} \frac{1}{4} \chi^{2} c^{2} b B_{2}^{(2 m+2)}}{s e_{2 m+1}^{\prime}(0, q) s e_{2 m+1}^{\prime}\left(\frac{\pi}{2}, q\right)} S E_{2 m+2}(v, q) \rightarrow J_{2 m+2}\left(\chi c v^{1 / 2}\right)
$$


(b) Consider the limit corresponding to the diagram $\lim _{e_{1} \rightarrow \infty}<e_{0} \mid e_{1}>\rightarrow$ $\left(e_{0}\right)$. Take the Mathieu equation as previously with $e_{0}=0$ and $e_{1}=b$, and the choice $v=b \sin ^{2} \theta$. With $a=\nu^{2}-\frac{1}{2} \chi^{2} c^{2} b, q=\frac{1}{4} \chi^{2} c^{2} b$ this equation assumes the form

$$
\left(\partial_{\theta}^{2}+a+2 q \cos \theta\right) \Phi=0 .
$$

In order to obtain a suitable limit as $b \rightarrow \infty$ we take $\nu^{2}=\left(2 n+\frac{1}{2}\right) b$ and $\chi^{2} c^{2}=\frac{b}{4}$. From these choices we see that if $v$ is fixed then $\theta$ is small and we have the relation $\theta=\sqrt{v / b}$. The corresponding limits can now be calculated:

$$
\frac{c e_{2 n}\left(\sqrt{\frac{v}{b}},-\frac{b}{16}\right)}{c e_{2 n}\left(0,-\frac{b}{16}\right)} \rightarrow \frac{\Gamma\left(\frac{1}{2}-n\right)}{\pi^{1 / 2} 2^{n}} D_{2 n}(\sqrt{v}), \frac{s e_{2 n+1}\left(\sqrt{\frac{v}{b}},-\frac{b}{16}\right)}{s e_{2 n+1}^{\prime}\left(0,-\frac{b}{16}\right)} \rightarrow-\frac{\Gamma\left(-\frac{1}{2}-n\right)}{\pi^{1 / 2} 2^{n+1}} D_{2 n+1}(\sqrt{v})
$$

where $D_{\nu}(z)$ is a parabolic cylinder function.

(c). Let us consider the limit corresponding to the diagram $\lim _{e_{0} \rightarrow \infty}<e_{0}^{2}>\rightarrow$ $\{\phi\}$. The separation equations for this coordinate system have the form

$$
\left\{4\left[\left(v-e_{0}\right)^{2} \partial_{v}^{2}+\left(v-e_{0}\right) \partial_{v}\right]-\nu^{2}+c^{2} \chi^{2}\left(v-e_{0}\right)\right\} \varphi=0,
$$

for $v=v_{1}, v_{2}$. In order to achieve this limit we can take

$$
e_{1} \rightarrow n^{2}, \quad c^{2} \rightarrow-n^{2}, \quad \nu^{2} \rightarrow \chi^{2} n^{4}+\beta n^{2} .
$$

The solutions for the function $\varphi$ are $C_{\nu}\left(\chi \sqrt{v-e_{0}}\right)$. The corresponding solutions for the limiting equation $\left[4 \partial_{v}^{2}+\chi^{2} v-\beta\right] \varphi=0$ are the Airy functions $A i\left[\left(\beta-\chi^{2} v\right) 2^{-2 / 3} \chi^{-4 / 3}\right]$ and $B i\left[\left(\beta-\chi^{2} v\right) 2^{-2 / 3} \chi^{-4 / 3}\right],[20]$. The limiting formula relating these solutions is

$$
A i\left[\frac{\beta}{\left(2 \chi^{2}\right)}\right] \frac{\pi\left(12 \chi^{2} n^{2}\right)^{\frac{1}{6}}}{\Gamma\left(\frac{1}{3}\right)} J_{\chi n^{2}+\frac{\beta}{2 \alpha}+\cdots}\left(-i \chi n \sqrt{v-n^{2}}\right) \rightarrow A i\left[\frac{\beta-\chi^{2} v}{\left(2 \alpha^{2}\right)^{\frac{2}{3}}}\right]
$$

as $n \rightarrow \infty$.

$(d)$. The last limiting process that we achieve can be represented as $\lim _{e_{0} \rightarrow \infty}\left(e_{0}\right) \rightarrow$ $\{\phi\}$. The separation equations for this coordinate system have the form

$$
\left\{4\left[\left(v-e_{0}\right) \partial_{v}^{2}+\frac{1}{2} \partial_{v}\right]+\lambda+c^{2} \chi^{2}\left(v-e_{0}\right)\right\} \varphi=0
$$

for $v=v_{1}, v_{2}$. In order to achieve this limit we can take

$$
e_{0} \rightarrow n^{2}, \quad \chi \rightarrow \sigma n, \quad \lambda \rightarrow \mu n^{2}+\sigma^{2} n^{4}
$$


The solutions for the function $\varphi$ are $D_{-\frac{i \lambda}{\chi}-\frac{1}{2}}\left( \pm 2 e^{i \pi / 4}\left[\chi\left(v-e_{0}\right)\right]^{1 / 2}\right)$. The corresponding solutions for the limiting equation $\left[4 \partial_{v}^{2}+\sigma^{2} v+\mu\right] \varphi=0$ are typically of the form $\left(\sigma^{2} v+\mu\right)^{1 / 2} C_{\frac{1}{3}}\left(\frac{2}{3} \frac{\left(\sigma^{2} v+\mu\right)^{\frac{3}{2}}}{\sigma^{2}}\right)$. Indeed the appropriate limit in this case is

$$
\begin{gathered}
\frac{2^{\frac{1}{2} i\left(\sigma n^{3}+\frac{\mu}{\sigma} n\right)+\frac{5}{2}}}{\frac{3}{4}-\frac{i}{2}\left(\sigma n^{3}+\frac{\mu}{\sigma} n\right)} D_{-i\left(\sigma n^{3}+\frac{\mu}{\sigma} n\right)-\frac{1}{2}}\left( \pm 2 e^{\frac{i \pi}{4}}\left[\sigma n\left(v-n^{2}\right)\right]^{\frac{1}{2}}\right) \rightarrow \\
\frac{\left(\sigma^{2} v+\mu\right)^{\frac{1}{2}} e^{i \frac{\pi}{6}}}{\sigma 3^{1 / 2}} H_{\frac{1}{3}}^{(1)}\left(\frac{2\left(\sigma^{2} v+\mu\right)^{\frac{3}{2}}}{3 \sigma^{2}}\right)
\end{gathered}
$$

as $n \rightarrow \infty$.

\section{References}

[1] E. Inönü and E. P. Wigner, On the contraction of groups and their representations, Proc. Nat. Acad. Sci. (US) 39, 510-524, (1953).

[2] J. Talman. Special Functions: A Group Theoretic Approach W.A. Benjamin, New York 1968.

[3] R. Gilmore, Lie Groups, Lie Algebras and Some of their Applications (Wiley, New York, 1974).

[4] A.Erdélyi, W.Magnus, F.Oberhettinger and F.G.Tricomi (eds) Higher Transcendental Functions, Vol. I,II McGraw Hill, New York, 1953.

[5] A.A.Izmest'ev, G.S.Pogosyan, A.N.Sissakian, and P.Winternitz. Contractions of Lie algebras and the separation of variables J.Phys.A, 29, 5940-5962 (1996).

[6] A.A.Izmest'ev, G.S.Pogosyan, A.N.Sissakian, and P.Winternitz. Contractions of Lie algebras and separation of variables. The twodimensional hyperboloid. Intl.J.Mod.Phys, A12, 53-61 (1997)

[7] A.A.Izmest'ev, G.S.Pogosyan, A.N.Sissakian, and P.Winternitz. Contractions of Lie algebras and separation of variables. The $N$-dimensional sphere, CRM-2527,1998 J. Math Phys., to appear , (1998). 
[8] E.G. Kalnins and W. Miller, Jr. Separation of variables on $n$-dimensional Riemannian manifolds I. The $n$-sphere and Euclidean $n$-space $J$. Math. Phys., 27, 1721, (1986).

[9] E.G. Kalnins. Separation of Variables for Riemannian Spaces of Constant Curvature, Pitman Monographs and Surveys in Pure and Applied Mathematics 28, Longman Scientific and Technical, Essex, England, 1986 .

[10] E.T. Whittaker and G.N. Watson. A Course of Modern Analysis (Chapter XXIII) Cambridge University Press, Cambridge, England 1973.

[11] E.G. Kalnins and W. Miller, Jr. Separable coordinates, integrability and the Niven equations J. Phys. A: Math. Gen., 25, 5663, (1992).

[12] P. Lancaster. Theory of Matrices (page 226) Academic Press, New York 1969.

[13] E.G. Kalnins, W. Miller and M.V. Tratnik. Families of orthogonal and biorthogonal polynomials on the $n$-sphere SIAM J. Math. Anal., 22, $272,(1991)$.

[14] E.G. Kalnins and W. Miller, Jr. Orthogonal polynomials on n-spheres: Gegenbauer, Jacobi and Heun, in Topics in Polynomials of One and Several Variables and their Applications: A Legacy of P.L. Chebyshev (1821-1894), H.M. Srivastava, T.M. Rassias and A. Yanushauskas, eds., World Scientific, Singapore, 1991.

[15] F. Arscott. Periodic Differential Equations. Macmillan, New York 1964.

[16] N.W. McLachlan. Theory and Application of Mathieu Functions Clarendon Press, Oxford, England 1947.

[17] L. Collatz. Functional Analysis and Numerical Mathematics (page 80) (translation from German) Academic Press, New York 1966.

[18] M.N.Olevskii. Triorthogonal Systems in Spaces of Constant Curvature in which the Equation $\Delta_{2} u+\lambda u=0$ Allows the Complete Separation of Variables Math.Sb., 27, 379 (1950) (in russian). 
[19] E.G. Kalnins, W. Miller Jr. and G.J. Reid. Separation of variables for complex Riemannian spaces of constant curvature 1. Orthogonal separable coordinates for coordinates $S_{n C}$ and $E_{n C}$ Proc. Roy. Soc. Lond., A394, 183, (1984).

[20] M.Abramowitz and I.A.Stegun. (eds) Handbook of Mathematical Functions (page 446), National Bureau of Standards Applied Math Series, Washington D.C., 1968. 